\title{
MONGE-AMPÈRE EQUATION AND BELLMAN OPTIMIZATION OF CARLESON EMBEDDING THEOREMS
}

\author{
VASILY VASYUNIN AND ALEXANDER VOLBERG
}

\begin{abstract}
Monge-Ampère equation plays an important part in Analysis. For example, it is instrumental in mass transport problems. On the other hand, the Bellman function technique appeared recently as a way to consider certain Harmonic Analysis problems as the problems of Stochastic Optimal Control. This brings us to Bellman PDE, which in stochastic setting is often a Monge-Ampère equation or its close relative. We explore the way of solving Monge-Ampère equation by a sort of method of characteristics to find the Bellman function of certain classical Harmonic Analysis problems, and, therefore, of finding full structure of sharp constants and extremal sequences for those problems.
\end{abstract}

\section{Introduction}

The goal of this article is to show how Monge-Ampère equation allows us to solve a certain class of Harmonic Analysis problems. We choose two problems to illustrate the method: John-Nirenberg inequality (JNI) and Carleson embeddding theorem (CET).

In these problems we determine Bellman function of the problem (see later). Bellman function carries all the information about the problem: sharp constant, construction of extremal functions/extremal sequences; and sometimes it also carries auxiliary information.

In both JNI and CET Bellman functions were found before: in [SV] for JNI and for CET in $[\mathrm{M}]$. However, the way they were found is rather specific for each problem and not "from basic principles" so-to-speak.

We propose here a universal method which fits many problems (we count at least seven of them). Among those we chose JNI and CET bacause of their classical nature and because the reader can easily compare our approach and our Bellman

1991 Mathematics Subject Classification. Primary: 28A80. Secondary: 28A75, 60D05.

Research of the authors was supported in part by NSF grants DMS-0501067 (Volberg) . 
functions with those in the literature $[\mathrm{SV}], \mathrm{M}]$. Our approach will differ completely from the previous ones, the functions will coincide.

We borrowed the Monge-Ampère solution by "the method of characteristics" from the literature, and the most important inspiration came from Slavin and Stokolos $\mathrm{SlSt}$.

Let us notice that the aim of the paper is not only to present a new and unified approach to finding Harmonic Analysis Bellman functions. In fact, for CET we plan to prove some new results, not just to reprove the old ones due to [M].

1.1. Bellman functions. The method of Bellman functions in Harmonic Analysis appeared probably in the 80's at the series of papers of Burkholder devoted to sharp $L^{p}$-estimates of martingale transform. It appeared there under certain disguise (and Bellman function and Bellman PDE were not even mentioned). The method was rediscovered in 1995 in the first preprint version of [NTV1]. It was very much developed in [NT]. The method turned out to be useful. Sometimes it solves certain important Harmonic Analysis problems that do not have an alternative (classical) approach so far, see e.g. [PVo. There is now certain amount of literature on Bellman function technique. We note here an excellent paper [M], which serves as our inspiration here, and two small surveys: [NTV2] and [Vo].

We fix $\varepsilon \in(0,1)$ and an interval $I$. Subintervals of $I$ will be called $J$. Here is what is called in [SV] the Bellman function for the John-Nirenberg inequality:

$$
\begin{aligned}
\mathbf{B}_{J N I}\left(x_{1}, x_{2} ; \varepsilon\right):=\sup \left\{\left\langle e^{\phi}\right\rangle_{I}:\langle\phi\rangle_{I}\right. & =x_{1},\left\langle\phi^{2}\right\rangle_{I}=x_{2} \\
& \left.\langle\phi\rangle_{J}^{2} \leq\left\langle\phi^{2}\right\rangle_{J} \leq\langle\phi\rangle_{J}^{2}+\varepsilon^{2} \quad \forall J \subset I\right\} .
\end{aligned}
$$

Function $\mathbf{B}_{J N I}$ is defined in

$$
\Omega_{J N I}:=\left\{x=\left(x_{1}, x_{2}\right): x_{1}^{2} \leq x_{2} \leq x_{1}^{2}+\varepsilon^{2}\right\} .
$$

Now given any $I$ we consider its dyadic lattice of subintervals $D=D(I)$ (it consists of left and right halves (sons) of $I$ and then all left and right grandsons and et cetera). Let $\mu$ denote a non-negative measure on $I$ without atoms. We want to write the Bellman function for the Carleson Embedding Theorem (dyadic). 


$$
\begin{gathered}
\mathbf{B}_{C E T}\left(x_{1}, x_{2}, x_{3} ; m, M\right):=\sup \frac{1}{|I|}\left\{\int_{I}|\phi(t)|^{2} d \mu(t)+\sum_{J \in D(I)}\left|\langle\phi\rangle_{J}\right|^{2} \alpha_{J}:\right. \\
\langle\phi\rangle_{I}=x_{1}, \quad\left\langle\phi^{2}\right\rangle_{I}=x_{2}, \quad \frac{1}{|I|}\left(\mu(I)+\sum_{J \in D(I)} \alpha_{J}\right)=x_{3}, \\
\left.m|J| \leq \mu(J)+\sum_{\ell \in D(J)} \alpha_{\ell} \leq M|J| \quad \forall J \in D(I)\right\} .
\end{gathered}
$$

Functions $\mathbf{B}_{C E T}$ is defined in

$$
\Omega_{C E T}:=\left\{x=\left(x_{1}, x_{2}, x_{3}\right): x_{1}^{2} \leq x_{2}, m \leq x_{3} \leq M\right\}
$$

We introduce also $\mathbf{B}\left(x_{1}, x_{2}, x_{3}\right)$. This is what is often called the Bellman function for the Carleson Embedding Theorem, see [M], [NT].

$$
\begin{aligned}
\mathbf{B}\left(x_{1}, x_{2}, x_{3}\right):=\sup \frac{1}{|I|}\left(\sum_{J \in D(I)}\left|\langle\phi\rangle_{J}\right|^{2} \alpha_{J}:\right. & \\
\langle\phi\rangle_{I}=x_{1}, \quad\left\langle\phi^{2}\right\rangle_{I}=x_{2}, \quad \frac{1}{|I|}\left(\sum_{J \in D(I)} \alpha_{J}\right)=x_{3}, & \left.\sum_{\ell \in D(J)} \alpha_{\ell} \leq|J| \quad \forall J \in D(I)\right\} .
\end{aligned}
$$

Remark. The reader can easily see that the definitions of all Bellman functions $\mathbf{B}_{C E T}, \mathbf{B}$ does not depend on the interval $I$.

The formula for the function $\mathbf{B}_{J N I}\left(x_{1}, x_{2}\right)$ was found in [V] and [SV], the formula for $\mathbf{B}\left(x_{1}, x_{2}, x_{3}\right)$ was found in $\left[\mathbf{M}\right.$, the general formula for $\mathbf{B}_{C E T}\left(x_{1}, x_{2}, x_{3} ; m, M\right)$ below is new. Let us also notice that formula for $\mathbf{B}\left(x_{1}, x_{2}, x_{3}\right)$ was found in [M] from intricate combinatorial consideration. We tried to explain in the present paper a natural way to find this formula from "the basic principles".

Namely, all formulae will be obtained here by a unified method of solving the Monge-Ampère equation.

\section{Theorem 1.}

$$
\mathbf{B}_{J N I}\left(x_{1}, x_{2} ; \varepsilon\right)=\frac{e^{-\varepsilon}}{1-\varepsilon}\left(1-\sqrt{\varepsilon^{2}-\left(x_{2}-x_{1}^{2}\right)}\right) e^{x_{1}+\sqrt{\varepsilon^{2}-\left(x_{2}-x_{1}^{2}\right)}} .
$$


Theorem 2. Consider

$$
B\left(x_{1}, x_{2}, x_{3}\right)=\frac{x_{3} x_{2}}{[1-2 a]^{2}\left[1-4 a\left(1-x_{3}\right)\right]},
$$

where $a=a(x)$ is the unique solution of the cubic equation

$$
\frac{x_{1}^{2}}{x_{2}}=\left[\frac{1-2 a\left(1-x_{3}\right)}{1-2 a}\right]^{2} \frac{1-4 a}{1-4 a\left(1-x_{3}\right)}
$$

on the interval $\left[0, \frac{1}{4}\right]$. Then

$$
\mathbf{B}(x)=B(x) \quad x_{1}^{2} \leq x_{2}, \quad 0<x_{3} \leq 1 .
$$

Theorem 3. Consider

$$
B\left(x_{1}, x_{2}, x_{3} ; m, M\right)=\frac{\left(x_{3}-m\right) x_{2}}{[1-2 a(M-m)]^{2}\left[1-4 a\left(M-x_{3}\right)\right]}+m x_{2},
$$

where $a=a(x)$ is the unique solution of the cubic equation

$$
\frac{x_{1}^{2}}{x_{2}}=\left[\frac{1-2 a\left(M-x_{3}\right)}{1-2 a(M-m)}\right]^{2} \frac{1-4 a(M-m)}{1-4 a\left(M-x_{3}\right)}
$$

on the interval $\left[0, \frac{1}{4(M-m)}\right]$. Then

$$
\mathbf{B}_{C E T}(x ; m, M)=\left\{\begin{array}{cll}
B(x ; m, M) & x_{1}^{2} \leq x_{2}, & m<x_{3} \leq M \\
m x_{2} & x_{1}^{2} \leq x_{2}, \quad x_{3}=m .
\end{array}\right.
$$

Remark. The cubic equation (1.9) has actually sometimes two solutions and sometimes one solution (for the left hand side in $[0,1)$ ). The one (called $a(x)$ above) is always in $\left[0, \frac{1}{4(M-m)}\right]$. Another one (if exists) is negative. We will call it $a^{-}=a^{-}(x)$. We will see now that it is also responsible for the meaningful and interesting extremal problem.

1.2. Lower Bellman function. If we denote the Bellman function (11.1) by $\mathbf{B}_{\max }$ and introduce another Bellman function

$$
\begin{gathered}
\mathbf{B}_{\min }\left(x_{1}, x_{2}, x_{3} ; m, M\right):=\inf \left\{\int_{I}|\phi(t)|^{2} d \mu(t)+\sum_{J \in D(I)}\left|\langle\phi\rangle_{J}\right|^{2} \alpha_{J}:\right. \\
\langle\phi\rangle_{I}=x_{1}, \quad\left\langle\phi^{2}\right\rangle_{I}=x_{2}, \quad \frac{1}{|I|}\left(\mu(I)+\sum_{J \in D(I)} \alpha_{J}\right)=x_{3}, \\
\left.m|J| \leq \mu(J)+\sum_{\ell \in D(J)} \alpha_{\ell} \leq M|J| \quad \forall J \in D(I)\right\},
\end{gathered}
$$


then we can show that $a=a^{-}$corresponds to this extremal problem.

Theorem 4. Consider

$$
B\left(x_{1}, x_{2}, x_{3} ; m, M\right)=\frac{\left(x_{3}-m\right) x_{2}}{[1-2 a(M-m)]^{2}\left[1-4 a\left(M-x_{3}\right)\right]}+m x_{2},
$$

where $a=a^{-}(x)$ is the unique negative solution of the cubic equation

$$
\frac{x_{1}^{2}}{x_{2}}=\left[\frac{1-2 a\left(M-x_{3}\right)}{1-2 a(M-m)}\right]^{2} \frac{1-4 a(M-m)}{1-4 a\left(M-x_{3}\right)} .
$$

Then

$$
\mathbf{B}_{\min }(x ; m, M)=\left\{\begin{array}{cc}
B(x ; m, M) & x_{1}^{2} \leq x_{2}, \quad M-(M-m) \frac{x_{1}^{2}}{x_{2}}<x_{3} \leq M \\
m x_{2} & x_{1}^{2} \leq x_{2}, \quad m \leq x_{3} \leq M-(M-m) \frac{x_{1}^{2}}{x_{2}} .
\end{array}\right.
$$

\section{Discussion.}

- 1. We are going to provide a detailed proof of Theorem 3 only. We leave for the reader to fill out the details of the proof of Theorem 4, where concavity should be repalced by convexity.

- 2. An interesting observation follows from the comparison of (1.6) and (1.8). In fact, we can see that the following rescaling relationship holds

$\mathbf{B}_{C E T}\left(x_{1}, x_{2}, x_{3} ; m, M\right)=(M-m) B\left(x_{1}, x_{2}, \frac{x_{3}-m}{M-m}\right)+m x_{2}$.

In principle, (1.12) should have followed just from the definitions of $\mathbf{B}$, $\mathbf{B}_{C E T}$ above. Let us provide a small explanation.

First, introduce the notations, by denoting $a_{l}$ to be the center of dyadic interval $l$, then

$$
c_{l}:=a_{l}+i \frac{|l|}{2}, d \alpha:=\sum_{l \in D(I)} \alpha_{l} d \delta_{c_{l}},
$$

where $\delta_{z}$ stands for the delta measure at $z$ as usual. Actually, (1.12) means that if we are forced to have the uniform estimate from below on how much measure we have in any closed Carleson square:

$$
(\mu+\alpha)\left(\bar{Q}_{J}\right) \geq m|J|, \forall J \in D(I),
$$

then to maximize the quantity in the definition of $\mathbf{B}_{C E T}(x ; m, M)$ over all admissible $\mu$ 's and $\alpha$ 's (that is to obtain $\mathbf{B}_{C E T}(x ; m, M)$ ) one needs to keep "boundary measure" $\mu$ to be exactly equal to $m d x$. In principle, it is 
easy to believe that always $\mu$ should be $\geq m d x$. But (1.12) claims more, namely, the equality. It means, that if we want to maximize the outcome in the definition of $\mathbf{B}_{C E T}(x ; m, M)$ we need to keep minimal possible mass (namely, $m d x$ ) on the boundary, as a reserve so-to-speak, and we need to distribute the rest of mass in the form of measure $\alpha$ somewhere inside the half-plane. Also (1.12) means that this distribution of $\alpha$ mimics and actually equals the rescaled distribution of the extremal measure $\alpha$ in the case when we maximize $\mathbf{B}$ and not $\mathbf{B}_{C E T}(x ; m, M)$.

- 3. Of course (1.12) shows that function $\mathbf{B}$ is "more equal" among $\mathbf{B}_{C E T}$. But interestingly enough, (1.12) seems not to follow directly from the definitions of functions $\mathbf{B}$ and $\mathbf{B}_{C E T}(x ; m, M)$ in (1.5) and (11.1) but rather from the proofs of Theorems 2 and 3 . In fact, it is a priori not clear why the optimal distribution of measure for $\mathbf{B}_{C E T}(x ; m, M)$ should have these two features: a) it leaves minimal possible "reserve" on the boundary of the circle; b) it distributes the rest by repeating the best distribution for B (with correctly rescaled parameters) as (1.12) shows.

We shall not prove Theorem [1, its proof can be found in [V] or [SV], in the latter paper the comparison of dyadic and non-dyadic cases for JNI can be found as well. We use this theorem as a "simple" example to illustrate how to find the Bellman function by using the general method of solving the Monge-Ampère equation. Then we use the same approach in much more involved situations of Theorem 3. This Bellman function is also not quite new, for the case $m=0, M=1$ the function was found by Melas in [M], where the reader can find the complete proof of the theorem for this the most important case.

However, in $\mathrm{M}$ there is no explanation how the author was able to guess his very non-trivial function. And the main accent of our paper is just the explanation how it is possible to guess right, or more correctly, how to solve the corresponding Bellman equation, which is a Monge-Ampère equation in the situation under consideration. Nevertheless, we will not stop after finding the Bellman function from Theorem 3 and provide the complete proof for the sake of completeness and the convenience of the reader and because in such more general setting it is new.

\section{HOW WE PROCEED?}

The consideration of the theorems above can be split to four parts. 
- I. In the first part one observes that just by definition Bellman functions B satisfy a certain concavity condition in their domain of definition and boundary conditions on (part of) the boundary of this domain.

- II. In the second part one considers all function satisfying these concavity and boundary conditions. We denote this class by $\mathcal{V}$. And one makes the following supposition: as $b$ belongs to $\mathcal{V}$ and is the "best" such function, it has to satisfy not only the concavity condition but also this concavity should become degenerate, i.e., the inequality has to turns into equality along some vector field on our domain $\Omega$. This brings to the picture the Monge-Ampère equation. One solves it using the boundary conditions mentioned above. The result is a function $B \in \mathcal{V}$. Function $B$ carries an interesting geometric information to be used later.

These two steps are in fact not necessary for the proof of the results, they are needed only to finding a function $B$, a candidate for a rôle of the Bellman function B. For example, in the excellent paper $[\mathrm{M}]$ very complicated Bellman functions appear as deus ex machina. As we shall see the analysis of Monge-Ampère equation not only supplies us with a candidate, but it helps in the next two steps as well, namely, in the prove that the found candidate really is the desired Bellman function.

- III. The third part consists of proving that $B \geq \mathbf{B}$. In convex domains of definition this is usually not difficult. Otherwise it may require a non-trivial proof, see $[\mathrm{SV}]$ for non-convex $\Omega_{\varepsilon}$.

- IV. The fourth part consists of proving $B \leq \mathbf{B}$. This is achieved by presenting the extremal functions or extremal sequences of functions. In its turn such functions are found from the geometric structure of $B$ (mentioned above in II).

\section{Monge-Ampère EquATion.}

This section is here for illustrative purposes. Through this section the function $\mathbf{B}$ is the Bellman function $\mathbf{B}_{J N I}$ for the John-Nirenberg inequality defined in (1.1) and $\Omega=\Omega_{J N I}$ is its domain defined in (1.2).

Let us very briefly review Part I for Theorem 1

It is very easy to see that in the dyadic case (i.e. in the case when only dyadic intervals $J \in D(I)$ are considered in (1.1) $)$ the function $\mathbf{B}$ has to satisfy concavity 
condition

$$
\mathbf{B}(x)-\frac{1}{2}\left(\mathbf{B}\left(x^{+}\right)+\mathbf{B}\left(x^{-}\right)\right) \geq 0,
$$

for all triples $x, x^{+}, x^{-}$such that $x=\frac{x^{+}+x^{-}}{2}$ and all three points are from $\Omega$.

In the non-dyadic case (i.e. in the case when all subintervals $J \subset I$ are considered in (1.1)) it is not clear a priori why the Bellman function $\mathbf{B}$ must satisfy the concavity condition (3.1). And it turns out that for the Bellman function condition (3.1) is not fulfilled. However, it turns out that the Bellman function is locally concave, i.e., concave in any convex subdomain of $\Omega$. We shall not discuss here this subtle moment, especially because we shall use just the local concavity condition, which is of course weaker than the global concavity, and coincides with the latter only for convex domains. The discussion of the difference between dyadic and non-dyadic Bellman functions for the John-Nirenberg inequality the reader can find in [SV]. Here we only add that for the functions smooth enough, the concavity condition can be rewritten in the differential form:

$$
d^{2} \mathbf{B}(x):=\left(\begin{array}{ll}
\mathbf{B}_{x_{1} x_{1}} & \mathbf{B}_{x_{1} x_{2}} \\
\mathbf{B}_{x_{2} x_{1}} & \mathbf{B}_{x_{2} x_{2}}
\end{array}\right) \leq 0, \quad \forall x \in \Omega,
$$

where by $\mathbf{B}_{x_{i} x_{j}}$ we denote the partial derivatives $\frac{\partial^{2} \mathbf{B}}{\partial x_{i} \partial x_{j}}$.

We have one obvious (from the definition) boundary condition on the lower parabola of the boundary $\partial \Omega$ :

$$
\mathbf{B}\left(x_{1}, x_{1}^{2}\right)=e^{x_{1}},
$$

because only the constant test functions $\phi$ correspond the points $x$ with $x_{2}=x_{1}^{2}$.

We also have a simple (from definition) homogeneity condition

$$
\mathbf{B}\left(x_{1}+t, x_{2}+2 x_{1} t+t^{2}\right)=e^{t} \mathbf{B}\left(x_{1}, x_{2}\right) .
$$

It follows from the definition of $\mathbf{B}$ if to take $\phi+t$ as a test function rather than $\phi$. Let us move to the Part II. Put

$$
\mathcal{V}=\left\{v \in C^{2}(\Omega): v \text { satisfies (3.2), (3.3), (3.4) }\right\} .
$$

Usually any function from $\mathcal{V}$ (and moreover, any function satisfying only the concavity condition (3.1) ) supply us with some estimate. But if we looked for a sharp estimate we need to choose the minimal possible function $v$ from this class, which must be the Bellman function B. This function, "a candidate in the 
true Bellman function" will be denoted by the usual letter $B$. For every point $x \in \Omega$ there exists an extremal function $\phi$ (or "almost extremal" function $\phi_{n}$, i.e., a sequence of functions) realizing the supremum in the definition (1.1). The usual procedure of using the Bellman function consists in the consecutive application of (3.1), when splitting the interval $I$, where a test function is defined. For the extremal test function there has to be no lost in such procedure, therefore, for the Bellman function the equality has to occur at least for one splitting the point $x$ into a pair $\left\{x^{+}, x^{-}\right\}$. For a concave function this means that it is linear at some direction. If we have almost extremal functions, i.e., an extremal sequence, then we must have "almost equality" in (3.1), at least up to the terms of second order. In any case this means that the Hessian matrix (3.2) has to be degenerated. Thus, we are looking for the "best" $B$, on the top of this condition of negativity of Hessian we will impose the following degeneration condition:

$$
\forall x \in \Omega \exists \Theta \in \mathbb{R}^{2} \backslash\{0\}:\left(\left(d^{2} B\right) \Theta, \Theta\right)=\left(\left(\begin{array}{ll}
B_{x_{1} x_{1}} & B_{x_{1} x_{2}} \\
B_{x_{2} x_{1}} & B_{x_{2} x_{2}}
\end{array}\right)\left(\begin{array}{c}
\Theta_{1} \\
\Theta_{2}
\end{array}\right),\left(\begin{array}{c}
\Theta_{1} \\
\Theta_{2}
\end{array}\right)\right)=0
$$

Since the matrix $d^{2} B$ is negatively defined, we conclude from (3.5) the following degeneration condition on the Hessian:

$$
\operatorname{det}\left(d^{2} B\right)=\operatorname{det}\left(\begin{array}{ll}
B_{x_{1} x_{1}} & B_{x_{1} x_{2}} \\
B_{x_{2} x_{1}} & B_{x_{2} x_{2}}
\end{array}\right)=0, \quad \forall x \in \Omega .
$$

We underline once more that in the first two steps we can allow ourself not too rigorous arguments and various assumptions, because this is not the proof, it is heuristic way of finding a candidate in the Bellman function. The rigorous proof that the found candidate is indeed the required Bellman function starts from the Part III.

Claim. There is a simple algorithm to find the family of functions enumerated by a parameter $\delta(\varepsilon \leq \delta \leq 1)$

$$
B=B_{\delta}\left(x_{1}, x_{2}\right):=\frac{e^{-\delta}}{1-\delta}\left(1-\sqrt{\delta^{2}-\left(x_{2}-x_{1}^{2}\right)}\right) e^{x_{1}+\sqrt{\delta^{2}-\left(x_{2}-x_{1}^{2}\right)}}
$$

that solves Monge-Ampère equation (3.6) with boundary condition (3.3) and homogeneity condition (3.4).

To prove this claim we need 
3.1. Monge-Ampère equation and method of "characteristics". Let $v=$ $v\left(x_{1}, \ldots, x_{n}\right)$ is a smooth function satisfying the following Monge-Ampère equation in some domain $\Omega$

$$
\operatorname{det} d^{2} v=\operatorname{det}\left(\begin{array}{ccc}
v_{x_{1} x_{1}} & \cdots & v_{x_{1} x_{n}} \\
\cdots & \cdots & \cdots \\
v_{x_{n} x_{1}} & \cdots & v_{x_{n} x_{n}}
\end{array}\right)=0, \quad \forall x=\left(x_{1}, \ldots, x_{n}\right) \in \Omega
$$

and suppose that this matrix has rank $n-1$, i.e., all smaller minors od $d^{2} v$ are non-zero. Then there are functions $t_{i}\left(x_{1}, \ldots, x_{n}\right), i=0,1, \ldots, n$, such that

$$
v(x)=t_{0}+t_{1} x_{1}+t_{2} x_{2}+\cdots+t_{n} x_{n}
$$

and the following $n-1$ linear equations hold:

$$
d t_{0}+x_{1} d t_{1}+x_{2} d t_{2}+\cdots+x_{n-1} d t_{n-1}+x_{n} d t_{n}=0 .
$$

Let us explain why this is $n-1$ equations and why they are linear. One needs to read (3.9) as follows: we think that, say, $t_{1}, \ldots, t_{n-1}$ are $n-1$ independent variables and $t_{n}, t_{0}$ are functions of them. Then (3.9) can be rewritten as follows

$$
\left(\frac{\partial t_{0}}{\partial t_{1}}+x_{1}+x_{n} \frac{\partial t_{n}}{\partial t_{1}}\right) d t_{1}+\cdots+\left(\frac{\partial t_{0}}{\partial t_{n-1}}+x_{n-1}+x_{n} \frac{\partial t_{n}}{\partial t_{n-1}}\right) d t_{n-1}=0
$$

whence

$$
x_{i}+x_{n} \frac{\partial t_{n}}{\partial t_{i}}+\frac{\partial t_{0}}{\partial t_{i}}=0, \quad i=1, \ldots, n-1 .
$$

So we get $n-1$ equations.

Remark. In general we can choose any $n-1$ variables as independent, of course. Since the order of variables is arbitrary, sometimes the first $n-1$ is not the most convenient choice.

Now why these are linear equations? We think that $t_{1}, \ldots, t_{n-1}$ is fixed. Then the $n-1$ equations give us linear relationships in $x_{1}, \ldots, x_{n}$, so $n-1$ hyperplanes.

Therefore, (3.9) gives the intersection of $n-1$ hyperplanes, so gives us a line. We can call it $L_{t_{1}, \ldots, t_{n-1}}$. These lines foliate domain $\Omega$ and (3.8) shows that $v$ is a linear function on each such line.

Let us prove all these propositions. Matrix $d^{2} v$ annihilates one vector $\Theta(x)$ at every $x=\left(x_{1}, \ldots, x_{n}\right) \in \Omega$. So we get a vector field $\Theta$. Consider its integral curve 
$x_{1}=x_{1}\left(x_{n}\right), \ldots, x_{n-1}=x_{n-1}\left(x_{n}\right)$. Vector $\Theta(x)$ is a tangent vector to that curve, i.e.,

$$
\Theta=\Theta_{n}\left(\begin{array}{c}
x_{1}^{\prime} \\
x_{2}^{\prime} \\
\cdots \\
x_{n-1}^{\prime} \\
1
\end{array}\right)
$$

Consider a new function $g\left(x_{n}\right)=v\left(x_{1}\left(x_{n}\right), \ldots, x_{n-1}\left(x_{n}\right), x_{n}\right)$. Due to (3.10) its second derivative is

$g^{\prime \prime}=\left\langle d^{2} v\left(\begin{array}{c}x_{1}^{\prime} \\ x_{2}^{\prime} \\ \cdots \\ x_{n-1}^{\prime} \\ 1\end{array}\right),\left(\begin{array}{c}x_{1}^{\prime} \\ x_{2}^{\prime} \\ \cdots \\ x_{n-1}^{\prime} \\ 1\end{array}\right)\right\rangle+v_{x_{1}} x_{1}^{\prime \prime}+\cdots+v_{x_{n-1}} x_{n-1}^{\prime \prime}=v_{x_{1}} x_{1}^{\prime \prime}+\cdots+v_{x_{n-1}} x_{n-1}^{\prime \prime}$.

Now, let us also show that $v_{x_{1}}, \ldots, v_{x_{n-1}}$ are constants on this integral curve. Suppose we are standing on the integral curve. The surface $v_{x_{1}}=t_{1}=$ const has normal $\left(v_{x_{1} x_{1}}, \ldots, v_{x_{1} x_{n}}\right)$, that is the first row of matrix $d^{2} v$, which is orthogonal to the directional vector $\Theta$ of the integral curve. Hence $\Theta$ is in the tangent hyperplane to the surface $v_{x_{1}}=t_{1}$. The same is true for the surfaces $v_{x_{i}}=t_{i}=$ const, $i=2, \ldots, n-1$. Intersection of these surfaces gives us our integral curves, because $\Theta$ is in the intersection of all tangent planes to these surfaces, therefore the curves $C_{t_{1}, \ldots, t_{n-1}}$ enumerated by constants $t_{1}, \ldots, t_{n}$ are just the integral curves of the tangent bundle $\Theta$. Thus, (3.11) can be rewritten as

$$
\frac{d^{2}}{d x_{n}^{2}}\left(g-\left(t_{1} x_{1}+\cdots+t_{n} x_{n-1}\right)\right)=0
$$

We obtain that the second derivative of a function (of $x_{n}$ ) in (3.12) is zero. So function is linear in $x_{n}$, that is $t_{n} x_{n}+t_{0}$, where the constants $t_{n}, t_{0}$ depend only on the curve $C_{t_{1}, \ldots, t_{n-1}}$, that is

$$
t_{n}=t_{n}\left(t_{1}, \ldots, t_{n-1}\right), \quad t_{0}=t_{0}\left(t_{1}, \ldots, t_{n-1}\right) .
$$

We obtain on $C_{t_{1}, \ldots, t_{n-1}}$

$$
v\left(x_{1}\left(x_{n}\right), \ldots, x_{n-1}\left(x_{n}\right), x_{n}\right)=t_{0}+x_{1} t_{1}+\cdots+x_{n-1} t_{n-1}+t_{n} x_{n} .
$$


Since we assumed our vector field to be smooth and its integral curves foliate the whole domain, varying parameters $t_{1}, \ldots, t_{n}$ we get (3.8). To check (3.9) take a full differential in (3.8). Then

$$
\left[v_{x_{1}} d x_{1}+\cdots+v_{x_{n}} d x_{n}\right]=d v=\left[d t_{0}+t_{1} d x_{1}+\cdots+t_{n} d x_{n}\right]+x_{1} d t_{1}+\cdots+x_{n} d t_{n} .
$$

We are on $C_{t_{1}, \ldots, t_{n}}$ and so $v_{x_{i}}=t_{i}, i=1, \ldots, n-1$ as we established already. But it is also easy to see that

$$
v_{x_{n}}=t_{n}\left(t_{1}, \ldots, t_{n-1}\right)
$$

on $C_{t_{1}, \ldots, t_{n-1}}$. In fact, all $v_{x_{i}}$ and all $t_{i}$ are symmetric. We could have chosen to represent the integral curve of $\Theta$ not as $x_{i}=x_{i}\left(x_{n}\right), i=1, \ldots, n-1$ but as $x_{j}=x_{j}\left(x_{1}\right), j=2, \ldots, n$. Now we see that two expression in brackets in (3.13) are equal. Then we obtain $x_{1} d t_{1}+\cdots+x_{n} d t_{n}+d t_{0}=0$, which is desired $n-1$ linear relationships (3.9).

3.2. The proof of the claim. We can use the previous Section 3.1. But we prefer to repeat it for the simple case of only two variables!

Proof. Equation (3.6) of course is solved by linear function in $x_{1}, x_{2}$, but linear function cannot satisfy the boundary condition. So we assume that matrix $d^{2} B$ is negatively defined, non-zero, and there exists exactly one vector $\Theta \in \mathbb{R}^{2} \backslash\{0\}$ that annihilates it: $d^{2} B \Theta=0$. This vector may depend of course on $\left(x_{1}, x_{2}\right)$ and we get a vector field $\Theta(x)$ in $\Omega$. Let us see now that $B_{x_{i}}, i=1,2$, are constants on the integral curves of this vector field. Locally our integral curves are level sets of a certain function $s=s\left(x_{1}, x_{2}\right)$. Of course, the function $s(x)$ is not uniquely defined, it is defined up to change of variables $s \mapsto \varphi \circ s$, where $\varphi$ is a function of one variable. But in any parametrization the vector $\left(-s_{x_{2}}, s_{x_{1}}\right)$ is a tangent vector to the integral curve, i.e., it is collinear with our vector field $\Theta$ and thus annihilates $d^{2} B$ :

$$
\left(\begin{array}{ll}
B_{x_{1} x_{1}} & B_{x_{1} x_{2}} \\
B_{x_{2} x_{1}} & B_{x_{2} x_{2}}
\end{array}\right)\left(\begin{array}{c}
-s_{x_{2}} \\
s_{x_{1}}
\end{array}\right)=0 .
$$

Notice that both functions $B_{x_{i}}\left(x_{1}, x_{2}\right)$ instead of $s\left(x_{1}, x_{2}\right)$ satisfy the last equation as well. In fact, the vector $\left(-\left(B_{x_{1}}\right)_{x_{2}},\left(B_{x_{1}}\right)_{x_{1}}\right)$ annihilates the first row of $d^{2} B$ obviously. Then it annihilates the second row just by (3.6). The same is true for the vector $\left(-\left(B_{x_{1}}\right)_{x_{2}},\left(B_{x_{1}}\right)_{x_{1}}\right)$. Therefore, the integral curves of our vector field are the level sets of the functions both $B_{x_{1}}\left(x_{1}, x_{2}\right)$ and $B_{x_{2}}\left(x_{1}, x_{2}\right)$. Thus, we can 
conclude that (at least locally) $B_{x_{i}}=t_{i} \circ s$ for some functions $t_{i}$ of one variable. Moreover, this means that we can take any of $B_{x_{i}}$ to play the role of $s$ : to define the integral curves of our vector field $\Theta$ as its level sets.

Let us check now that the function $t_{0}:=B-t_{1} x_{1}-t_{2} x_{2}$ is also constant along any integral curve. For this aim we have to check that we can put $t_{0}$ instead of $s$ into (3.14), but it is evident, because at each point $\left(x_{1}, x_{2}\right)$ we have

$$
\begin{aligned}
-\frac{\partial t_{0}}{\partial x_{2}} & =-\frac{\partial B}{\partial x_{2}}+\frac{\partial t_{1}}{\partial x_{2}} x_{1}+\frac{\partial t_{2}}{\partial x_{2}} x_{2}+t_{2}=B_{x_{1} x_{2}} x_{1}+B_{x_{2} x_{2}} x_{2} \\
\frac{\partial t_{0}}{\partial x_{1}} & =\frac{\partial B}{\partial x_{1}}-\frac{\partial t_{1}}{\partial x_{1}} x_{1}-\frac{\partial t_{2}}{\partial x_{1}} x_{1}-t_{1}=-B_{x_{1} x_{1}} x_{1}-B_{x_{2} x_{1}} x_{2}
\end{aligned}
$$

So, we obtained

$$
B(x)=t_{0}+t_{1} x_{1}+t_{2} x_{2} .
$$

The integral curve of the kernel field of the Hessian of this function are the straight lines given by the equation

$$
d t_{0}+x_{1} d t_{1}+x_{2} d t_{2}=0 .
$$

Indeed, on the one hand

$$
d B=B_{x_{1}} d x_{1}+B_{x_{2}} d x_{2}
$$

on the other hand the differential of (3.15) is

$$
d B=d t_{0}+x_{1} d t_{1}+t_{1} d x_{1}+x_{2} d t_{2}+t_{2} d x_{2} .
$$

Comparison of the these two equalities yields (3.16).

If the vertical integral curves of our vector field are nowhere dense (so they could be given by $\left.x_{2}=x_{2}\left(x_{1}\right)\right)$, then they could be considered as the level sets $t_{2}=t$, and two other parameters are functions of $t$. Equations (3.15) and (3.16) could be rewritten as

$$
B(x)=t_{0}(t)+t_{1}(t) x_{1}+t x_{2}, \quad t_{0}(t)^{\prime}+t_{1}(t)^{\prime} x_{1}+x_{2}=0 .
$$

If the horizontal integral curves of our vector field are nowhere dense, they could be written by $x_{1}=x_{1}\left(x_{2}\right)$, or as the level sets $t_{1}=t$, and two other parameters are functions of $t$. Equations (3.15) and (3.16) then takes the form

$$
B(x)=t_{0}(t)+t x_{1}+t_{2}(t) x_{2}, \quad t_{0}(t)^{\prime}+x_{1}+t_{2}(t)^{\prime} x_{2}=0 .
$$


Till now we have considered general equation when the determinant of the Hessian for a function of two variables is zero. Now we return to our specific problem and use (3.4) to determine the structure of the family of integral lines.

Differentiating (3.4) in $t$ and setting $t=0$ we get

$$
B_{x_{1}}+2 x_{1} B_{x_{2}}=B
$$

or

$$
t_{1}+2 x_{1} t_{2}=t_{0}+t_{1} x_{1}+t_{2} x_{2} .
$$

This is the equation of a straight line

$$
\left(t_{0}-t_{1}\right)+x_{1}\left(t_{1}-2 t_{2}\right)+x_{2} t_{2}=0
$$

representing a level set of the functions $t_{i}$, therefore it has to be the same straight line that is given by equation (3.16). Hence the following two equations must be true

They could be rewritten as

$$
\frac{d t_{0}}{t_{0}-t_{1}}=\frac{d t_{1}}{t_{1}-2 t_{2}}=\frac{d t_{2}}{t_{2}}
$$

$$
\begin{gathered}
d\left(\frac{t_{0}}{t_{2}}\right)=-t_{1} \frac{d t_{2}}{t_{2}^{2}} \\
d\left(\frac{t_{1}}{t_{2}}\right)=-2 \frac{d t_{2}}{t_{2}} .
\end{gathered}
$$

Solution of (3.21) is

$$
t_{1}=-2 t_{2} \log \left|t_{2}\right|+2 c_{1} t_{2}
$$

(we denoted the integration constant by $2 c_{1}$ for the future convenience). After plugging this solution into (3.20), we get

$$
t_{0}=t_{2} \log ^{2}\left|t_{2}\right|-2 c_{1} t_{2} \log \left|t_{2}\right|+c_{2} t_{2} .
$$

Now we rewrite equation (3.19) using the obtained expressions for $t_{i}$ :

$$
\left(\log ^{2}\left|t_{2}\right|+2\left(1-c_{1}\right) \log \left|t_{2}\right|+\left(c_{2}-2 c_{1}\right)\right)-2 x_{1}\left(\log \left|t_{2}\right|-c_{1}+1\right)+x_{2}=0 .
$$

It is convenient introduce a new variable

$$
a:=\log \left|t_{2}\right|-c_{1}+1 .
$$

Then the formulas (3.22) and (3.23) can be rewritten as follows:

$$
\begin{aligned}
& t_{1}=2(1-a) t_{2}, \\
& t_{0}=\left((1-a)^{2}-c_{1}^{2}+c_{2}\right) t_{2},
\end{aligned}
$$


and the extremal lines are

$$
x_{2}-2 a x_{1}+a^{2}+c_{2}-1-c_{1}^{2}=0 .
$$

Since

$$
c_{1}^{2}+1-c_{2}=x_{2}-2 a x_{1}+a^{2}=\left(x_{2}-x_{1}^{2}\right)+\left(x_{1}-a\right)^{2} \geq 0,
$$

we can introduce a new non-negative constant $\delta$ such that

$$
\delta^{2}=c_{1}^{2}+1-c_{2}
$$

and rewrite equation (3.26) in the form

$$
x_{2}-2 a x_{1}+a^{2}-\delta^{2}=0 .
$$

We observe that these are lines tangent to parabola $x_{2}=x_{1}^{2}+\delta^{2}$ at the point $x_{1}=a, x_{2}=a^{2}+\delta^{2}$ and intersecting the lower boundary $x_{2}=x_{1}^{2}$ at the points with $x_{1}=a \pm \delta$. We want these lines to foliate domain $\Omega=\left\{\left(x_{1}, x_{2}\right): x_{1}^{2} \leq x_{2} \leq x_{1}^{2}+\varepsilon^{2}\right\}$. Hence,

$$
\delta \geq \varepsilon
$$

Now we can almost write down $B\left(x_{1}, x_{2}\right)$. The variable $a$ can be found in terms of $x_{i}$ by solving equation (3.27):

$$
a=x_{1}+\sqrt{\delta^{2}-\left(x_{2}-x_{1}^{2}\right)} .
$$

We have chosen the solution with the plus sign in front of the square root. The explanation, why does it correspond to our problem, and to what extremal problem corresponds the opposite sign, can be found in [V, SV]. Using now (3.24), (3.25), and (3.27) we obtain the expression for $B$ :

$$
\begin{aligned}
B\left(x_{1}, x_{2}\right) & =t_{0}+t_{1} x_{1}+t_{2} x_{2} \\
& =\left((1-a)^{2}-c_{1}^{2}+c_{2}+2(1-a) x_{1}+x_{2}\right) t_{2} \\
& =2\left(1-a+x_{1}\right) t_{2}= \pm 2\left(1-a+x_{1}\right) e^{a+c_{1}-1} \\
& = \pm 2\left(1-\sqrt{\delta^{2}-\left(x_{2}-x_{1}^{2}\right)}\right) e^{x_{1}+\sqrt{\delta^{2}-\left(x_{2}-x_{1}^{2}\right)}+c_{1}-1} .
\end{aligned}
$$

Since $B$ has to be positive and to satisfy boundary condition (3.3) we choose the sign and the value of the constant $c_{1}$. Finally, we get a family of solutions

$$
B\left(x_{1}, x_{2} ; \delta\right)=\frac{1-\sqrt{\delta^{2}-\left(x_{2}-x_{1}^{2}\right)}}{1-\delta} e^{x_{1}+\sqrt{\delta^{2}-\left(x_{2}-x_{1}^{2}\right)}-\delta}
$$

depending on a parameter $\delta$. Acceptable values of the parameter $\delta$ are $0<\delta<1$. Why it is so, as well as the choice of this parameter for a given $\epsilon$ (by the way, 
different for the dyadic and non-dyadic cases), an explanation of all these facts can be found in [SV].

The above family of functions $B$ was found in [V] and [SV] from different reasoning. After finding this candidate the steps I, II are completely finished for John-Nirenberg inequality. The reader is referred to [SV], where steps III and IV are done. The fact that $\Omega$ is not convex makes step III rather delicate.

Now we are going to present for the reader all four steps for another more difficult problem of finding the Bellman functions of Carleson embedding theorems (CET).

\section{Step I FOR CARLESON EMBEDDing TheOREMS}

From the definition of function $\mathbf{B}$ above we can immediately see that it satisfies the inequality (just concavity)

$$
\mathbf{B}(x)-\frac{1}{2}\left(\mathbf{B}\left(x^{-}\right)+\mathbf{B}\left(x^{+}\right)\right) \geq 0, \quad \forall x^{ \pm} \in \Omega, \quad x=\frac{1}{2}\left(x^{+}+x^{-}\right) .
$$

Note that now $\Omega=\Omega_{C E T}$ (see (1.4) ) is convex and for all $x^{ \pm} \in \Omega$ we have $x \in \Omega$.

For the sake of brevity, we shall usually omit the parameters $m$ and $M$.

The boundary conditions also follow from the definition:

$$
\begin{gathered}
\mathbf{B}\left(x_{1}, x_{1}^{2}, x_{3}\right)=x_{1}^{2} x_{3} . \\
\frac{\partial \mathbf{B}}{\partial x_{3}}\left(x_{1}, x_{2}, M ; m, M\right)=x_{1}^{2} .
\end{gathered}
$$

It is equally easy to see that homogeneity condition holds

$$
\mathbf{B}\left(t x_{1}, t^{2} x_{2}, x_{3}\right)=t^{2} \mathbf{B}\left(x_{1}, x_{2}, x_{3}\right), t \in \mathbb{R} .
$$

The detailed explanation of these properties can be found in [NT], where this Bellman function was defined and a majorant (so called supersolution) was found.

Let us consider all smooth functions in $\Omega$ satisfying (4.1), (4.2), (4.4). Call this family $\mathcal{V}$.

As before, we are looking for the "best" $B \in \mathcal{V}$, so on the top of the condition (4.1) of negativity of Hessian we will impose the following degeneration condition: 


$$
\begin{aligned}
\forall x=\left(x_{1}, x_{2}, x_{3}\right) \in \Omega \quad \exists \Theta \in \mathbb{R}^{3} \backslash\{0\} \\
\left(\left(d^{2} B\right) \Theta, \Theta\right)=\left(\left(\begin{array}{lll}
B_{x_{1} x_{1}} & B_{x_{1} x_{2}} & B_{x_{1} x_{3}} \\
B_{x_{2} x_{1}} & B_{x_{2} x_{2}} & B_{x_{2} x_{3}} \\
B_{x_{3} x_{1}} & B_{x_{3} x_{2}} & B_{x_{3} x_{3}}
\end{array}\right)\left(\begin{array}{c}
\Theta_{1} \\
\Theta_{2} \\
\Theta_{3}
\end{array}\right),\left(\begin{array}{c}
\Theta_{1} \\
\Theta_{2} \\
\Theta_{3}
\end{array}\right)\right)=0 .
\end{aligned}
$$

As matrix $d^{2} B$ is negatively defined we conclude from (3.5) the following degeneration condition on the Hessian:

$$
\operatorname{det}\left(d^{2} B\right)=\operatorname{det}\left(\begin{array}{lll}
B_{x_{1} x_{1}} & B_{x_{1} x_{2}} & B_{x_{1} x_{3}} \\
B_{x_{2} x_{1}} & B_{x_{2} x_{2}} & B_{x_{2} x_{3}} \\
B_{x_{3} x_{1}} & B_{x_{3} x_{2}} & B_{x_{3} x_{3}}
\end{array}\right)=0, \quad \forall x \in \Omega
$$

Claim. There is a simple algorithm to find the function $B(x ; m, M)$ that solves Monge-Ampère equation (4.6) in the domain (1.4) with boundary conditions (4.2)(4.3) and homogeneity condition (4.4).

Proof. Again we consider the vector field $\Theta$ such that $d^{2} B(x) \Theta(x)=0, x=$ $\left(x_{1}, x_{2}, x_{3}\right) \in \Omega$. And we consider its integral curves. Section 3.1 shows that these integral curves are straight lines (segments of straight lines). Section 3.1 and its the method of characteristics allows us to write the following "parametric" equation for these lines, which we first write in the invariant form (compare with (3.16) )

$$
x_{1} d t_{1}+x_{2} d t_{2}+x_{3} d t_{3}+d t_{0}=0 .
$$

This can be rewritten as follows if we choose $\left(t_{1}, t_{2}\right)$ as the set of independent parameters defining lines $L_{t_{1}, t_{2}}$ foliating our domain:

$$
\left\{\begin{array}{l}
x_{1}+\frac{\partial t_{3}}{\partial t_{1}} \cdot x_{3}+\frac{\partial t_{0}}{\partial t_{1}}=0 \\
x_{2}+\frac{\partial t_{3}}{\partial t_{2}} \cdot x_{3}+\frac{\partial t_{0}}{\partial t_{2}}=0
\end{array}\right.
$$

And similarly to the previous section the solution of the Monge-Ampère equation is given by

$$
B\left(x_{1}, x_{2}, x_{3}\right)=t_{1} \cdot x_{1}+t_{2} \cdot x_{2}+t_{3} \cdot x_{3}+t_{0} .
$$


Here each line is given by fixing two free parameters $\left(t_{1}, t_{2}\right)$, which are $t_{1}=\frac{\partial B}{\partial x_{1}}$, $t_{2}=\frac{\partial B}{\partial x_{2}}$. (This can be obtained exactly as in the previous section.) Parameters $t_{3}, t_{0}$ are not free, they are unknown functions of $t_{1}, t_{2}$, for which we will find (a priori non-linear) PDE. They will be easy in our case (and linear). And we will solve them easily. This will bring us the formula for $B$ in the same way we get when proving the claim of Section 3 ,

Let us use (4.4) now. Differentiating in $t$ and setting $t=1$ we get

$$
x_{1} t_{1}+2 x_{2} t_{2}=2 B \text {. }
$$

Whence,

$$
t_{1} x_{1}+2 t_{3} x_{3}+2 t_{0}=0 \text {. }
$$

Homogeneity gave us (4.11) and we conclude that this plane (for each fixed $t_{1}$, $t_{2}$ ) contains the line (4.8). But the line (4.8) is passing through the point

$$
\left(-\frac{\partial t_{0}}{\partial t_{1}},-\frac{\partial t_{0}}{\partial t_{2}}, 0\right)
$$

and has the direction

$$
\left(-\frac{\partial t_{3}}{\partial t_{1}},-\frac{\partial t_{3}}{\partial t_{2}}, 1\right)
$$

Hence we get from (4.11):

$$
\left\{\begin{array}{l}
2 t_{3}-t_{1} \frac{\partial t_{3}}{\partial t_{1}}=0 \\
2 t_{0}-t_{1} \frac{\partial t_{0}}{\partial t_{1}}=0
\end{array}\right.
$$

From these "PDE" we easily write down

$$
\left\{\begin{array}{l}
t_{3}=A\left(t_{2}\right) t_{1}^{2} \\
t_{0}=D\left(t_{2}\right) t_{1}^{2}
\end{array}\right.
$$

Then the equations of the extremal lines $L_{t_{1}, t_{2}}$ can be rewritten in the form

$$
\left\{\begin{array}{l}
x_{1}+2 t_{1} A x_{3}+2 t_{1} D=0 \\
x_{2}+t_{1}^{2} A^{\prime} x_{3}+t_{1}^{2} D^{\prime}=0
\end{array}\right.
$$

We need to work a bit to define functions $A$ and $D$. We assume that our line intersects $\partial \Omega$ in a point $\zeta=\zeta\left(t_{1}, t_{2}\right)$ on the "upper lid" $x_{3}=M$ and in a point 
$\xi=\xi\left(t_{1}, t_{2}\right)$ on the "side" $x_{2}=x_{1}^{2}$. Then we have two pairs of equations (4.16) asserting that our points are on the line $L_{t_{1}, t_{2}}$

$$
\begin{array}{r}
\zeta_{1}+2 t_{1} A M+2 t_{1} D=0 \\
\zeta_{2}+t_{1}^{2} A^{\prime} M+t_{1}^{2} D^{\prime}=0 \\
\xi_{1}+2 t_{1} A \xi_{3}+2 t_{1} D=0 \\
\xi_{1}^{2}+t_{1}^{2} A^{\prime} \xi_{3}+t_{1}^{2} D^{\prime}=0
\end{array}
$$

and two boundary conditions (4.2) and (4.3)

$$
\begin{aligned}
\frac{1}{2} \xi_{1} t_{1}+\xi_{1}^{2} t_{2} & =\xi_{1}^{2} \xi_{3} \\
A t_{1}^{2} & =\zeta_{1}^{2} .
\end{aligned}
$$

Thus, we have six equations with six unknown functions: $\zeta_{1}, \zeta_{2}, \xi_{1}, \xi_{3}, A$, and $D$. Equation (4.18) determines the function $\zeta_{2}$. If we take $\zeta_{1}$ from (4.17) and plug into (4.22) we get

$$
A=4(A M+D)^{2} .
$$

So, introducing a new function of $t_{2}$

$$
a=2(A M+D)
$$

we can express both functions $A$ and $D$ in terms of $a$ : 4.23) yields

$$
A=a^{2}
$$

and directly from the definition of $a$ (4.24) we get

$$
D=\frac{1}{2} a-M a^{2} \text {. }
$$

Note that deducing (4.23) we divided both parts of (4.22) over $t_{1}$. This is a correct operation because the Bellman function $\mathbf{B}$ clearly depends on all variables $x_{i}$, and therefore its partial derivatives $t_{i}$ cannot be identically zero. By the way, since $t_{3}$ is not identically zero, so is $a$. Moreover, we can assert that $a$ is not a constant function. Indeed, assuming $a$ to be a constant, we have $\zeta_{2}=0$ from (4.18), whence $\zeta_{1}=0$ due to $\zeta_{1}^{2} \leq \zeta_{2}=0$. But (4.17) can be written as $\zeta_{1}+a t_{1}=0$, and since neither $t_{1}$ nor $a$ is not zero, we come to a contradiction.

In result we reduce our system to the system of three equations with three unknown functions $a\left(t_{2}\right), \xi_{1}\left(t_{1}, t_{2}\right)$, and $\xi_{3}\left(t_{1}, t_{2}\right)$. Indeed, equations (4.19), (4.20), 
and (4.21) can be rewritten as follows

$$
\begin{aligned}
\xi_{1}+t_{1} a\left(1-2 a\left(M-\xi_{3}\right)\right) & =0 \\
\xi_{1}^{2}+t_{1}^{2} a^{\prime}\left(\frac{1}{2}-2 a\left(M-\xi_{3}\right)\right) & =0 \\
\frac{1}{2} \xi_{1} t_{1}-\xi_{1}^{2}\left(\xi_{3}-t_{2}\right) & =0 .
\end{aligned}
$$

To have possibility divide (4.29) over $\xi_{1}$, we need to check that $\xi_{1}=0$ cannot be an appropriate solution. Assuming that identically $\xi_{1}=0$ we get $1-2 a\left(M-\xi_{3}\right)=0$ from (4.27), which turns (4.28) into $-\frac{1}{2} t_{1}^{2} a^{\prime}=0$. But this is impossible because, $t_{1} \neq 0$ and $a$ is not a constant function. So, we can rewrite (4.29) as

$$
\xi_{3}=t_{2}+\frac{t_{1}}{2 \xi_{1}}
$$

In fact $\xi_{3}$ does not depend on $t_{1}$. Indeed, if we introduce

$$
\eta=1-2 a\left(M-\xi_{3}\right),
$$

equations (4.27) and (4.28) turn into

$$
\begin{aligned}
\xi_{1}+t_{1} a \eta & =0 \\
\xi_{1}^{2}+t_{1}^{2} a^{\prime}\left(\eta-\frac{1}{2}\right) & =0,
\end{aligned}
$$

whence

$$
a^{\prime}\left(\eta-\frac{1}{2}\right)+a^{2} \eta^{2}=0 .
$$

Since $a$ does not depend on $t_{1}$, the function $\eta$ (as a solution of this equation) also depends only on $t_{2}$. Soon we shall show that $x_{3}$ does not depend on $t_{2}$ either, it is a constant function!

But now we remove $\xi_{3}$ temporary from the play. From (4.31) we have

$$
\xi_{3}=M-\frac{1-\eta}{2 a},
$$

and (4.30) together with (4.32) yield

$$
\xi_{3}=t_{2}-\frac{1}{2 a \eta}
$$

The resulting equation is

$$
t_{2}-\frac{1}{2 a \eta}=M-\frac{1-\eta}{2 a},
$$

or

$$
t_{2}-M=\frac{\eta^{2}-\eta+1}{2 a \eta} .
$$


In result, we have two equations (4.34) and (4.37) for two unknown functions $a$ and $\eta$ of the variable $t_{2}$. Of course, now we could solve quadratic equation (4.34) with respect to $\eta$ and plug the solution in (4.37) trying to solve the resulting differential equation with respect to $a$. This is possible, but it leads to many complications. Much simpler is to differentiate (4.37)

$$
1=\frac{a \eta^{\prime}\left(\eta^{2}-1\right)-a^{\prime}\left(\eta^{3}-\eta^{2}+\eta\right)}{2 a^{2} \eta^{2}}
$$

and then to replace the denominator by using (4.34)

$$
a^{\prime}(2 \eta-1)=a \eta^{\prime}\left(\eta^{2}-1\right)-a^{\prime}\left(\eta^{3}-\eta^{2}+\eta\right)
$$

or

$$
\left(\eta^{2}-1\right)(\eta-1) a^{\prime}=a\left(\eta^{2}-1\right) \eta^{\prime} .
$$

First we consider the possible constant solutions $\eta\left(t_{2}\right)= \pm 1$. Solution $\eta=1$ is not suitable. Indeed, if $\eta=1$, then from (4.37) we get

$$
a=\frac{1}{2\left(t_{2}-M\right)},
$$

whence

$$
\begin{gathered}
A=a^{2}=\frac{1}{4\left(t_{2}-M\right)^{2}}, D=\frac{1}{2} a-M a^{2}=\frac{t_{2}-2 M}{4\left(t_{2}-M\right)^{2}}, \\
A^{\prime}=-\frac{1}{2\left(t_{2}-M\right)^{3}}, D^{\prime}=-\frac{t_{2}-3 M}{4\left(t_{2}-M\right)^{3}} .
\end{gathered}
$$

Therefore, in this case, the extremal line $L_{t_{1}, t_{2}}$ (cf. (4.16) has the form

$$
\left\{\begin{array}{l}
x_{1}+\frac{t_{1} x_{3}}{2\left(t_{2}-M\right)^{2}}+t_{1} \frac{t_{2}-2 M}{2\left(t_{2}-M\right)^{2}}=0 \\
x_{2}-\frac{t_{1}^{2} x_{3}}{2\left(t_{2}-M\right)^{3}}-t_{1}^{2} \frac{t_{2}-3 M}{4\left(t_{2}-M\right)^{3}}=0 .
\end{array}\right.
$$

But the only point of this line with $x_{3}=M$ belongs to our domain $\Omega$, because for other points we have

$$
x_{2}-x_{1}^{2}=\frac{t_{1}^{2}\left(2 x_{3}+t_{2}-3 M\right)}{4\left(t_{2}-M\right)^{3}}-\frac{t_{1}^{2}\left(x_{3}+t_{2}-2 M\right)^{2}}{4\left(t_{2}-M\right)^{4}}=-\frac{t_{1}^{2}\left(x_{3}-M\right)^{2}}{4\left(t_{2}-M\right)^{4}}<0 .
$$

Solution $\eta=-1$ is also impossible. Therefore, we come to

$$
(\eta-1) a^{\prime}=a \eta^{\prime}
$$

which implies $a=C \cdot(\eta-1)$. Then (4.35) yields

$$
\xi_{3}\left(t_{2}\right)=M+\frac{1}{2 C}=: c .
$$


Recall that $\xi_{3}\left(t_{1}, t_{2}\right)=c$ is the level on which the line $L_{t_{1}, t_{2}}$ intersects the boundary $x_{2}=x_{1}^{2}, 0 \leq x_{3} \leq 1$, i.e., the third coordinate of the point of intersection. So, $m \leq c \leq M$. By our assumptions these lines intersect the boundary $x_{3}=M$, therefore, to foliate the whole domain $\Omega$ (at least its interior) we need to have $c=m$. So, from now on we assume $\xi_{3}=c=m$. Of course, we could consider any $c>m$ and try to find an additional solution of the Monge-Ampére equation in the subdomain $m \leq x_{3} \leq c$ but we shall not do this. First of all, we do not need to look for another solution. The better way of doing is to try now to check that the found function is just the Bellman function of the problem. Would we have some obstacles in proving we could continue our search. Another argument explaining why we do not need "to glue" our candidate from two parts is the fact that there is no concave solution in the subdomain $m \leq x_{3} \leq c$ being continuous extension of the found solution.

Recall that we have the following expression for $B$ (see (4.10) )

$$
B=\frac{1}{2} t_{1} x_{1}+t_{2} x_{2} .
$$

Thus, to find an expression for $B$ we need to find $t_{1}$ and $t_{2}$ as functions of a point $x$ running over our domain $\Omega$.

From (4.36) we have

$$
t_{2}=m+\frac{1}{2 a[1-2 a(M-m)]} .
$$

This gives us the desired $a$ as a function of $t_{2}$, but it is clear that $a$ is more convenient parameter than $t_{2}$. We shall express all other functions of $t_{2}$ in terms of $a$ and look for $a$ as a function of $x \in \Omega$. To this aim we return to the equations of the extremal lines (4.16) rewriting them in terms of $a$. From (4.44) we have

$$
\frac{d t_{2}}{d a}=-\frac{1-4 a(M-m)}{2 a^{2}[1-2 a(M-m)]^{2}}
$$

therefore

$$
a^{\prime}=-\frac{2 a^{2}[1-2 a(M-m)]^{2}}{1-4 a(M-m)}
$$

and

$$
\begin{gathered}
A=a^{2}, A^{\prime}=2 a a^{\prime}=-\frac{4 a^{3}[1-2 a(M-m)]^{2}}{1-4 a(M-m)}, \\
D=\frac{1}{2} a-M a^{2}, D^{\prime}=\frac{1}{2} a^{\prime}(1-4 a M)=-\frac{a^{2}(1-4 a M)[1-2 a(M-m)]^{2}}{1-4 a(M-m)} .
\end{gathered}
$$


Now equation (4.16) of the line $L_{t_{1}, t_{2}}$ becomes

$$
\left\{\begin{array}{l}
x_{1}=-t_{1} a\left[1-2 a\left(M-x_{3}\right)\right], \\
x_{2}=t_{1}^{2} a^{2} \frac{[1-2 a(M-m)]^{2}\left[1-4 a\left(M-x_{3}\right)\right]}{1-4 a(M-m)} .
\end{array}\right.
$$

As we have known, the side surface $\left\{x_{2}=x_{1}^{2}\right\}$ is intersected by $L_{t_{1}, t_{2}}$ at the points of the "bottom lid" $\left\{x_{3}=m\right\}$

$$
\xi=\left(\xi_{1}, \xi_{1}^{2}, m\right), \quad \xi_{1}=-t_{1} a[1-2 a(M-m)] .
$$

If we take $\xi_{1}$ for parameterizing the extremal lines, then their equations will be more symmetric. Indeed, (4.49) implies

$$
t_{1}=-\frac{\xi_{1}}{1+2 a(M-m)}
$$

and therefore (4.48) turns into

$$
\left\{\begin{array}{l}
x_{1}=\frac{1-2 a\left(M-x_{3}\right)}{1-2 a(M-m)} \xi_{1} \\
x_{2}=\frac{1-4 a\left(M-x_{3}\right)}{1-4 a(M-m)} \xi_{1}^{2} .
\end{array}\right.
$$

These equations immediately supply us with an expression for $a$, namely, $a=a(x)$ is a root of the following cubic equation

$$
s:=\frac{x_{1}^{2}}{x_{2}}=\left[\frac{1-2 a\left(M-x_{3}\right)}{1-2 a(M-m)}\right]^{2} \frac{1-4 a(M-m)}{1-4 a\left(M-x_{3}\right)} .
$$

To determine, which of three possible roots of the equation (4.52) gives us the desired value of $a$, we investigate the above function $s=s(a)$ defined by (4.52) as a function of the parameter $a$, all other parameters assuming to be fixed.

First of all we note that the extremal line (4.51) intersects the plane $\left\{x_{3}=M\right\}$ at the point

$$
\zeta=\left(\zeta_{1}, \zeta_{2}, M\right), \quad \zeta_{1}=\frac{\xi_{1}}{1-2 a(M-m)}, \quad \zeta_{2}=\frac{\xi_{1}^{2}}{1-4 a(M-m)} .
$$

Since $\zeta_{2} \geq 0$, we have the first restriction for $a$ :

$$
a<\frac{1}{4(M-m)} .
$$

The behavior of $s(a)$ on the semi-axis (4.54) is the same for all values of other parameters: on the negative half-line it monotonously increases from $\frac{M-x_{3}}{M-m}$ till 1 , and on the interval $\left[0, \frac{1}{4(M-m)}\right]$ it monotonously decreases from 1 to 0 . Therefore, 
for a given $s, 0 \leq s \leq 1$, we have one or two solutions of (4.52) satisfying (4.54): for all $s$ there exists a positive solution $a=a^{+}$and for $s>\frac{M-x_{3}}{M-m}$ there exists a negative solution $a=a^{-}$as well. We shall see that our solution is $a=a^{+}$, but the solution $a=a^{-}$is not meaningless: unexpectedly we get a solution of another extremal problem, namely (1.10).

To complete step II of constructing a Bellman function candidate we need to write down formula (1.8) or (1.11). Everything is ready to do this: we have expression (4.43) for $B$, where we need to substitute (4.44) for $t_{2}$ and to take $t_{1}$ from the first line of (4.48). So, we get

$$
\begin{aligned}
B(x ; m, M) & =-\frac{x_{1}^{2}}{2 a\left[1-2 a\left(M-x_{3}\right)\right]}+\left(m+\frac{1}{2 a[1-2 a(M-m)]}\right) x_{2} \\
& =\frac{\left(x_{3}-m\right) x_{2}}{[1-2 a(M-m)]^{2}\left[1-4 a\left(M-x_{3}\right)\right]}+m x_{2},
\end{aligned}
$$

where $a=a^{+}$is the positive root $\left(4(M-m) a^{+} \leq 1\right)$ of the cubic equation (4.52) for Theorem [3, and $a=a^{-}$is the negative one for Theorem 4 ,

\section{Foliation according to $B$.}

We already saw that lines $L_{t_{1}, t_{2}}$ are given by equations (4.51) and in the parametrization of the family of the extremal lines it is more convenient to use parameter $a$ instead of $t_{2}$ and $\xi_{1}$ instead of $t_{1}$ :

$$
\ell_{a, \xi_{1}}:=L_{t_{1}, t_{2}}
$$

For a given $\xi_{1}$ we have a "fan" of extremal lines starting at an arbitrary point $\xi=\left(\xi_{1}, \xi_{1}^{2}, m\right)$ on the "edge" $\left\{x: x_{2}=x_{1}^{2}, x_{3}=m\right\}$ :

$$
\begin{aligned}
& F_{\xi_{1}}^{+}=\left\{\ell_{a, \xi_{1}}: 0 \leq a<\frac{1}{4(M-m)}\right\}, \\
& F_{\xi_{1}}^{-}=\left\{\ell_{a, \xi_{1}}:-\infty<a \leq 0\right\},
\end{aligned}
$$

where $F_{\xi_{1}}^{+}$is the fan of extremal lines of $B_{\max }$ and $F_{\xi_{1}}^{-}$is the fan of extremal lines of $B_{\min }$. 
Let us find the trace of our fan on the boundary $x_{3}=M$. We remember that the point of intersection $\zeta$ is given by (4.53):

$$
\left\{\begin{array}{l}
\zeta_{1}=\frac{\xi_{1}}{1-2 a(M-m)}, \\
\zeta_{2}=\frac{\xi_{1}^{2}}{1-4 a(M-m)} .
\end{array}\right.
$$

This is a parametric equations of the hyperbola $C_{\xi_{1}}$

$$
\zeta_{2}=\frac{\zeta_{1} \xi_{1}^{2}}{2 \xi_{1}-\zeta_{1}}
$$

tangent to the parabola $\zeta_{2}=\zeta_{1}^{2}$ at the point $\left(\xi_{1}, \xi_{1}^{2}\right)$.

The intersection of the fan $F_{\xi_{1}}^{+}$with the plane $x_{3}=M$ is the piece $C_{\xi_{1}}^{+}$of this hyperbola between the points $\xi_{1}$ and $2 \xi_{1}$, for the fan $F_{\xi_{1}}^{-}$it is the piece $C_{\xi_{1}}^{-}$of this hyperbola between $\xi_{1}$ and zero. For $\xi_{1}=0$ it is the axis $C_{0}^{ \pm}=\left\{\left(0, \zeta_{2}\right)\right\}$.

Everything is on the "upper lid" $\left\{x_{3}=M, x_{1}^{2} \leq x_{2}\right\}$ of $\Omega$, and the curves $C_{x_{1}}^{+}$, as well as the curves $C_{x_{1}}^{-}$, foliate this "upper lid". Lines $\ell_{a, \xi_{1}} \in F_{\xi_{1}}^{ \pm}$connect the points of $C_{\xi_{1}}^{ \pm}$to the point $\left(\xi_{1}, \xi_{1}^{2}, m\right)$ on the boundary of $\Omega$ and each of two sets of lines $\left\{F_{\xi_{1}}^{ \pm}:-\infty<\xi_{1}<+\infty\right\}$ foliates some subdomain of $\Omega$. The lines of the fan $F_{\xi_{1}}^{+}$foliate the whole $\Omega$, whereas $F_{\xi_{1}}^{-}$foliate the subdomain

$$
\left\{x=\left\{x_{1}, x_{2}, x_{3}\right\}: \frac{M-x_{3}}{M-m}<\frac{x_{1}^{2}}{x_{2}} \leq 1, x_{3} \leq M\right\},
$$

where the function $B_{\min }$ is defined. Indeed, we have a solution $a=a^{+}$of (4.52) for arbitrary point of $\Omega$, while a solution $a=a^{-}$exists if and only if the point $x$ is from (5.2).

\section{LOWER BOUNDARY OF $\Omega$.}

Notice that we found our function $B$ only in $\left\{x_{1}^{2} \leq x_{2}, m<x_{3} \leq M\right\}$ for $B_{\max }$ and in $\left\{x_{1}^{2} \leq x_{2}, M-(M-m) \frac{x_{1}^{2}}{x_{2}}<x_{3} \leq M\right\}$ for $B_{\min }$. However both these functions are continuous in the closed domains. For $B_{\min }$ clearly the limit is $m x_{2}$ as $x_{3} \rightarrow M-(M-m) \frac{x_{1}^{2}}{x_{2}}$, what corresponds to $a \rightarrow-\infty$. For $B_{\max }$ formula (4.56) has indeterminancy $\frac{0}{0}$ when $x_{3} \rightarrow m$, what corresponds $a \rightarrow \frac{1}{4(M-m)}$. But we can easily pass to the limit in (4.55) and obtain by continuity the values of the function $B_{\max }\left(x_{1}, x_{2}, m ; m, M\right)$ on the "lower lid". Namely,

$$
B_{\max }\left(x_{1}, x_{2}, m ; m, M\right)=4(M-m)\left(x_{2}-x_{1}^{2}\right)+m x_{2} .
$$


The norm of embedding becomes apparent. In fact, the best constant in the inequality

$$
B_{\max } \leq C x_{2}
$$

can be read from (6.1), and it is indeed $4(M-m)$. This can be easily proved, but notice that it is not even explicit in the formula for $B_{\max }$ !

\section{REDUCING PARAMETERS $M$ AND $m$.}

Before we start to prove Theorems 3 and 4 , we would like to show that it is sufficient to prove them for $m=0$ and $M=1$. On the one hand, we have

$$
\mathbf{B}\left(x_{1}, x_{2}, x_{3} ; m, M\right)=\mathbf{B}\left(x_{1}, x_{2}, \frac{x_{3}-m}{M-m} ; 0,1\right)+m x_{2} .
$$

This is direct consequence of definition. Indeed, while the homogeneity condition (4.4) follows from the definition if to compare a set of test functions $\left\{\phi:\langle\phi\rangle_{I}=\right.$ $\left.x_{1},\left\langle\phi^{2}\right\rangle_{I}=x_{2}\right\}$ and the set $\{\tilde{\phi}=t \phi\}$ with the same measure $\mu$ and the same set of point masses $\alpha_{J}$, relation (7.1) follows from considering the same set of test functions $\phi$ but comparing with a "renormalized" measure $\tilde{\mu}(J)=\frac{\mu(J)-m|J|}{M-m}$ and point masses $\tilde{\alpha}_{J}=\frac{\alpha_{J}}{M-m}$, then we shall have $\tilde{m}=0, \tilde{M}=1$, and $\tilde{x}_{3}=\frac{x_{3}-m}{M-m}$.

Notice that function $\mathbf{B}(x)$ from (1.5) is defined differently than $\mathbf{B}_{C E T}(x ; 0,1)$. Its definition does not allow for measure $\mu$ on the boundary. However, these functions turned out to be equal, extremal $\mu$ must be zero. It is not clear how to see this immediately from the definitions (1.5) and (11.1) of $\mathbf{B}(x)$ and $\mathbf{B}_{C E T}(x ; 0,1)$ correspondingly. However, since the supremum in the definition of $\mathbf{B}(x)$ is taken over the smaller set of test measures $(\mu=0)$, we have the inequality $\mathbf{B}(x) \leq$ $\mathbf{B}_{C E T}(x ; 0,1)$. So, the proof of Theorems 2 and 3 will consist in proving two inequalities: $\mathbf{B}_{C E T}(x ; 0,1) \leq B(x ; 0,1)$ and $\mathbf{B}(x) \geq B(x ; 0,1)$.

Let us introduce the notations:

$$
B_{c}(x):=B(x ; 1-c, 1), \mathbf{B}_{c}(x):=\mathbf{B}_{C E T}(x ; 1-c, 1) c \in(0,1] .
$$

Obviously we can rescale everything and consider only $B_{c}$ and $\mathbf{B}_{c}$. Moreover, it is enough to consider only $c=1$.

In fact, our Bellman function candidate $B(x ; m, M)$ clearly satisfies relation (7.1), therefore, to prove $\mathbf{B}_{C E T}(x ; m, M)=B(x ; m, M)$ it is sufficient to check that

$$
\mathbf{B}_{C E T}(x ; 0,1)=B(x ; 0,1)
$$


for all suitable arguments $x$. We will first prove that

$$
B(x) \geq \mathbf{B}(x ; 0,1) .
$$

Then we prove

$$
B \leq \mathbf{B}
$$

Then the obvious inequality

$$
\mathbf{B} \leq \mathbf{B}(x ; 0,1)
$$

finishes the proof.

\section{8. $B_{c}(x) \geq \mathbf{B}_{c}(x)$. Concavity.}

In what follows symbol $B(x)$ stands always for $B(x ; 0,1)$ from (1.6) (which is the same as $B_{c}(x)$ with $\left.c=1\right)$.

Theorem 5. Function $B_{c}$ is concave in the domain $\Omega_{c}$.

Proof. It is enough to prove this for $c=1$. Then rescaling proves the rest. In what follows $a$ is always the unique root of (4.52) (with $M=1, m=0$ ) lying in $\left[0, \frac{1}{4}\right.$ ). In previous sections we calculated $(c=1)$ :

$$
\begin{array}{r}
\frac{\partial B}{\partial x_{1}}=t_{1}=-\frac{x_{1}}{a\left[1-2 a\left(1-x_{3}\right)\right]} \\
\frac{\partial B}{\partial x_{2}}=t_{2}=\frac{1}{2 a(1-2 c a)} \\
\frac{\partial B}{\partial x_{3}}=t_{3}=\frac{x_{1}^{2}}{\left[1-2 a\left(1-x_{3}\right)\right]} .
\end{array}
$$

We can now compute Hessian $d^{2} B$, where (we use the notation $s=x_{1}^{2} / x_{2}$ )

$$
\begin{gathered}
d^{2} B(x):=M(x)= \\
{\left[\begin{array}{ccc}
-\frac{1}{a\left[1-2 a\left(1-x_{3}\right)\right]}+\frac{1-4 a\left(1-x_{3}\right)}{a^{2}\left[1-2 a\left(1-x_{3}\right)\right]^{2}} \cdot \frac{2 x_{1}^{2}}{x_{2}} \cdot \frac{\partial a}{\partial s}, & -\frac{1-4 a}{a^{2}(1-2 a)^{2}} \cdot \frac{x_{1}}{x_{2}} \cdot \frac{\partial a}{\partial s}, & -\frac{2 x_{1}}{\left[1-2 a\left(1-x_{3}\right)\right]^{2}}-\frac{x_{1}\left[1-4 a\left(1-x_{3}\right)\right]}{a^{2}\left[1-2 a\left(1-x_{3}\right)\right]^{2}} \cdot \frac{\partial a}{\partial x_{3}} \\
-\frac{1-4 a\left(1-x_{3}\right)}{a^{2}\left[1-2 a\left(1-x_{3}\right)\right]^{2}} \cdot \frac{x_{1}^{3}}{x_{2}^{2}} \cdot \frac{\partial a}{\partial s}, & \frac{1-4 a}{2 a^{2}(1-2 a)^{2}} \cdot \frac{x_{1}^{2}}{x_{2}^{2}} \cdot \frac{\partial a}{\partial s}, & -\frac{1-4 a}{2 a^{2}(1-2 a)^{2}} \cdot \frac{\partial a}{\partial x_{3}} \\
\frac{2 x_{1}}{\left[1-2 a\left(1-x_{3}\right)\right]^{2}}+\frac{1-x_{3}}{\left[1-2 a\left(1-x_{3}\right)\right]^{3}} \cdot \frac{8 x_{1}^{3}}{x_{2}} \frac{\partial a}{\partial s} & -\frac{1-x_{3}}{\left[1-2 a\left(1-x_{3}\right)\right]^{3}} \cdot \frac{4 x_{1}^{4}}{x_{2}^{2}} \cdot \frac{\partial a}{\partial x_{3}} & -\frac{4 x_{1}^{2} a}{\left[1-2 a\left(1-x_{3}\right)\right]^{3}}+\frac{4 x_{1}^{2}\left(1-x_{3}\right)}{\left[1-2 a\left(1-x_{3}\right)\right]^{3}} \cdot \frac{\partial a}{\partial x_{3}}
\end{array}\right]}
\end{gathered}
$$

The element $M_{12}$ actually is equal to $M_{21}, M_{13}$ is equal to $M_{31}$ by (4.52). The reader may try to prove that this matrix is non-positive for every $x \in \Omega$ by direct calculation. We prefer an oblique way of doing that. Put 


$$
\begin{gathered}
N(x):=\left[\begin{array}{cc}
-\frac{1}{a\left[1-2 a\left(1-x_{3}\right)\right]}+\frac{1-4 a\left(1-x_{3}\right)}{a^{2}\left[1-2 a\left(1-x_{3}\right)\right]^{2}} \cdot \frac{2 x_{1}^{2}}{x_{2}} \cdot \frac{\partial a}{\partial s}, & -\frac{1-4 a}{a^{2}(1-2 a)^{2}} \cdot \frac{x_{1}}{x_{2}} \cdot \frac{\partial a}{\partial s} \\
-\frac{1-4 a\left(1-x_{3}\right)}{a^{2}\left[1-2 a\left(1-x_{3}\right)\right]^{2}} \cdot \frac{x_{1}^{3}}{x_{2}^{2}} \cdot \frac{\partial a}{\partial s}, & \frac{1-4 a}{2 a^{2}(1-2 a)^{2}} \cdot \frac{x_{1}^{2}}{x_{2}^{2}} \cdot \frac{\partial a}{\partial s}
\end{array}\right] \\
L(x):=\left[\begin{array}{ccc}
\frac{1-4 a\left(1-x_{3}\right)}{a^{2}\left[1-2 a\left(1-x_{3}\right)\right]^{2}} \cdot \frac{2 x_{1}^{2}}{x_{2}} \cdot \frac{\partial a}{\partial s}, & -\frac{1-4 a}{a^{2}(1-2 a)^{2}} \cdot \frac{x_{1}}{x_{2}} \cdot \frac{\partial a}{\partial s} \\
-\frac{1-4 a\left(1-x_{3}\right)}{a^{2}\left[1-2 a\left(1-x_{3}\right)\right]^{2}} \cdot \frac{x_{1}^{3}}{x_{2}^{2}} \cdot \frac{\partial a}{\partial s}, & \frac{1-4 a}{2 a^{2}(1-2 a)^{2}} \cdot \frac{x_{1}^{2}}{x_{2}^{2}} \cdot \frac{\partial a}{\partial s}
\end{array}\right]
\end{gathered}
$$

Let us prove that

$$
M_{11}<0, L \leq 0, N \leq 0
$$

We know that function $s \rightarrow a(s)$ decreases from $\frac{1}{4}$ to 0 when $s$ goes from 0 to 1 . Therefore, $a^{\prime} \geq 0$. Also $1-4 a\left(1-x_{3}\right)=(1-4 a)+4 a x_{3} \geq 0$ because $a \leq \frac{1}{4}, x_{3} \geq 0$, and $a>0$. Therefore, $L_{11} \leq 0, N_{22}=L_{22} \leq 0, N_{11}<0$ for every $x \in \Omega \cap\left\{0<x_{3}\right\}$. In particular, $M_{11}<0$.

On the other hand, it is immediate to see that $L_{11} L_{22}-L_{12} L_{21}$ vanishes identically. Then we can see right away that $N \leq 0$ as well, but more than that

$$
N<0, \operatorname{det} N(x) \neq 0 \quad \forall x \in \Omega \cap\left\{0<x_{3}\right\} \cap\left\{x_{1} \neq 0\right\} .
$$

In fact, it follows because $N=L+\operatorname{diag}\left\{-\frac{1}{a\left[1-2 a\left(1-x_{3}\right)\right]}, 0\right\}$ if we notice that

$$
-\frac{1}{a\left[1-2 a\left(1-x_{3}\right)\right]}<0
$$

and $L_{22} \leq 0$.

We would like to recall the reader that we obtained $B$ of (4.55)-(4.56) just by solving the Monge-Ampère equation $\operatorname{det} d^{2} B=0$ in $\Omega$. So, of course, $\operatorname{det} M(x)=0$. However, we propose to the reader to check this as follows.

Fix a point $x \in \Omega, 0<x_{3}$, and consider the line $L_{t_{1}, t_{2}}$ passing through $x$. Its directional vector was computed, it is (see (4.13), (4.15), (4.46), and (4.48) combined):

$$
d(x)=\left(\frac{2 a x_{1}}{1-2 a\left(1-x_{3}\right)} ; \frac{4 a(1-2 a)^{2} x_{1}^{2}}{(1-4 a)\left[1-2 a\left(1-x_{3}\right)\right]^{2}} ; 1\right)^{T} .
$$

Actually we built $B$ from the condition that $d^{2} B$ annihilate the vector field $d(x), x \in \Omega$. Also it is easy to see that $M(x) d(x)=0$ from the direct calculation. 
We saw that for every $x \in \Omega \cap\left\{0<x_{3}\right\}$ we have $M_{11}<0, M_{11} M_{22}-M_{12}^{2} \leq$ 0 , $\operatorname{det} M(x)=0$. By a well-known fact from linear algebra we conclude that $d^{2} B=$ $M(x)$ is negatively defined for all such $x$. Concavity of $B$ is fully proved.

Theorem 6. In $\Omega$ we have $\frac{\partial B}{\partial x_{3}}(x) \geq x_{1}^{2}$. The same is true for $B_{c}$ in $\Omega_{c}$ for $c \in(0,1]$.

Proof. The second claim follows from the first by rescaling. The first claim is obvious from (8.1) $: \frac{\partial B}{\partial x_{3}}=\frac{x_{1}^{2}}{\left[1-2 a\left(1-x_{3}\right)\right]^{2}} \geq x_{1}^{2}$ because $0<1-2 a\left(1-x_{3}\right) \leq 1$. We already noted the left inequality, it follows from $a>0$ and $a \leq \frac{1}{4} \leq \frac{1}{2}$. The right inequality is just $a>0$.

Theorem 7. Let $c \in(0,1]$. In $\Omega_{c}$ we have $\mathbf{B}_{c} \leq B_{c}$.

Proof. Again it is enough to consider only the case $c=1$. Let us combine Theorem 5 and Theorem 6 to obtain the following main inequality:

$$
\begin{array}{r}
B\left(x_{1}, x_{2}, x_{3}\right)-\frac{1}{2}\left[B\left(x_{1}^{+}, x_{2}^{+}, x_{3}^{+}\right)+B\left(x_{1}^{-}, x_{2}^{-}, x_{3}^{-}\right)\right] \geq x_{1}^{2}\left[x_{3}-\frac{1}{2}\left(x_{3}^{+}+x_{3}^{-}\right)\right], \\
\forall x, x^{+}, x^{-} \in \Omega \text { such that } x_{1}=\frac{1}{2}\left(x_{1}^{+}+x_{1}^{-}\right), x_{2}=\frac{1}{2}\left(x_{2}^{+}+x_{2}^{-}\right), \\
x_{3}=\frac{1}{2}\left(x_{3}^{+}+x_{3}^{-}\right) .
\end{array}
$$

In fact, this is easy. Put $x_{i}(t)=\frac{1}{2}\left[x_{i}^{+}(1+t)+x_{i}^{-}(1-t)\right], i=1,2$, and

$$
x_{3}(t)=\left\{\begin{array}{l}
-t x_{3}^{-}+x_{3}(1+t), \text { if } t \in[-1,0], \\
x_{3}(1-t)+t x_{3}^{+}, \text {if } t \in[0,1] .
\end{array}\right.
$$

Set

$$
b(t):=B\left(x_{1}(t), x_{2}(t), x_{3}(t)\right), t \in[-1,1] .
$$

Then the main inequality above transforms into

$$
b(0)-\frac{1}{2}\left(b(1)+b(-1) \geq x_{1}(0)^{2}\left[x_{3}(0)-\frac{1}{2}\left(x_{3}(1)+x_{3}(-1)\right] .\right.\right.
$$


To prove (8.9) let us notice that $x_{3}(t)$ is concave, and negative measure $x_{3}^{\prime \prime}$ is the following $x_{3}^{\prime \prime}(t)=-2\left[x_{3}(0)-\frac{1}{2}\left(x_{3}(1)+x_{3}(-1)\right] \cdot \delta_{0}\right.$. Then, of course, $b$ is concave (see Theorems 5 and 6) and measure

$$
\begin{gathered}
b^{\prime \prime}(t)=\left(d^{2} B x^{\prime}(t), x^{\prime}(t)\right) d t+\frac{\partial B}{\partial x_{3}}(x(t)) x_{3}^{\prime \prime}(t)=\left(d^{2} B(x(t)) x^{\prime}(t), x^{\prime}(t)\right) d t \\
-2 \frac{\partial B}{\partial x_{3}}(x(0))\left[x_{3}(0)-\frac{1}{2}\left(x_{3}(1)+x_{3}(-1)\right] \delta_{0} .\right.
\end{gathered}
$$

The following formula finishes the proof:

$$
b(0)-\frac{1}{2}\left(b(1)+b(-1)=-\frac{1}{2} \int_{-1}^{1}(1-|t|) b^{\prime \prime}(t) .\right.
$$

In fact, combining the last two formulae we obtain (we use Theorems 5 and [6again)

$$
\begin{aligned}
b(0)-\frac{1}{2}(b(1)+b(-1)= & \frac{1}{2} \int_{-1}^{1}(1-|t|)\left(-d^{2} B(x(t)) x^{\prime}(t), x^{\prime}(t)\right) d t \\
+ & \frac{\partial B}{\partial x_{3}}(x(0))\left[x_{3}(0)-\frac{1}{2}\left(x_{3}(1)+x_{3}(-1)\right]\right. \\
& \geq x_{1}(0)^{2}\left[x_{3}(0)-\frac{1}{2}\left(x_{3}(1)+x_{3}(-1)\right],\right.
\end{aligned}
$$

which is desired (8.9).

Now the main inequality $(8.6)$ and the convexity of the domain $\Omega$ will allow us to finish the proof of the theorem. We need a simple lemma about subharmonic functions on graphs.

We consider the dyadic tree $\mathcal{T}$, whose vertices are denoted by $p_{\sigma}$, where $\sigma$ is the word formed by \pm , the empty word $\sigma_{0}$ is the root of the tree, and $|\sigma|$ is the length of the word. Function on $\mathcal{T}$ is called superharmonic if its discrete Laplacian is non-negative

$$
\forall \sigma \in \mathcal{T} \Delta f(\sigma)=f(\sigma)-\frac{1}{2}(f(\sigma+)+f(\sigma-)) \geq 0 .
$$

We can associate the boundary of the tree with segment $[0,1]$ in the following sense. For Lebesgue almost every point $x \in[0,1]$ we have a unique branch $b(x)=$ $\left(\sigma_{0}, \sigma_{1}(x), \ldots, \sigma_{n}(x), \ldots\right)$ of $\mathcal{T}$ associated with it: just consider the dyadic form of $x$ and its $n$-th digit encodes whether to branch to + or - side on the $n$-th stage.

Given $f$ on the tree we put

$$
F(x)=\liminf _{n \rightarrow \infty} f\left(\sigma_{n}(x)\right) .
$$


Here is a lemma which deserves to be called Green's formula for the dyadic tree

Lemma 8. Given a positive finite superharmonic function $f$ on $\mathcal{T}$ we get

$$
\int_{0}^{1} F(x) d x+\sum_{\sigma \in \mathcal{T}} 2^{-|\sigma|} \Delta f(\sigma) \leq f\left(\sigma_{0}\right) .
$$

Proof. Obvious.

Now fix $x=\left(x_{1}, x_{2}, x_{3}\right) \in \Omega$ and fix any function $\phi$ on the interval $I=[0,1]$ (we recall that nothing depends on the original interval) with

$$
\langle\phi\rangle_{I}=x_{1},\left\langle\phi^{2}\right\rangle_{I}=x_{2},
$$

a non-negative measure $\mu$ on $I$, such that $0 \leq \mu(J) \leq|J|$ for all dyadic subintervals $J$ of $I$, and any collection of non-negative numbers $\left\{\alpha_{J}\right\}_{J \in D(I)}$ such that

$$
\frac{\mu(I)}{|I|}+\frac{1}{|I|} \sum_{J \in D(I)} \alpha_{J}=x_{3} \in(0,1], \frac{\mu(\ell)}{|\ell|}+\frac{1}{|\ell|} \sum_{J \in D(\ell)} \alpha_{J} \in[0,1] \forall \ell \in D(I) .
$$

We immediately see that $\mu=w d x$, where $0 \leq w \leq 1$.

Intervals of $D(I)$ and dyadic tree $\mathcal{T}$ are in natural one to one correspondence. We call $I_{\sigma}$ the interval corresponding to vertex $\sigma, I$ corresponds to $\sigma_{0}$. Consider the following function. Take $B$ from (4.55) (with $M=1, m=0$ ) and put

$$
M(\sigma):=\frac{1}{\left|I_{\sigma}\right|}\left(\mu\left(I_{\sigma}\right)+\sum_{J \in D\left(I_{\sigma}\right)} \alpha_{J}\right)
$$

and

$$
f(\sigma):=B\left(\langle\phi\rangle_{I_{\sigma}},\left\langle\phi^{2}\right\rangle_{I_{\sigma}}, M(\sigma)\right)
$$

Then of course

$$
f\left(\sigma_{0}\right)=B\left(x_{1}, x_{2}, x_{3}\right) .
$$

By the main inequality (8.6) it is a superharmonic function on the tree $\mathcal{T}$ : more than that, we can estimate its discrete Laplacian from below. By this same (8.6)

$$
\Delta f(\sigma) \geq\langle\phi\rangle_{I_{\sigma}}^{2} \Delta M(\sigma) .
$$

It is immediately seen that

$$
\Delta M(\sigma)=\alpha_{I_{\sigma}} /\left|I_{\sigma}\right| \geq 0 .
$$


Therefore, combining the last two inequalities we get

$$
\frac{2^{|\sigma|}\langle\phi\rangle_{I_{\sigma}}^{2} \alpha_{I_{\sigma}}}{\left|I_{\sigma_{0}}\right|} \leq \Delta f(\sigma)
$$

One can observe that $B\left(x_{1}, x_{2}, x_{3}\right) \geq x_{2} x_{3}$. Then

$$
f(\sigma) \geq\left\langle\phi^{2}\right\rangle_{I_{\sigma}} M(\sigma) \geq\left\langle\phi^{2}\right\rangle_{I_{\sigma}} \cdot \frac{\mu\left(I_{\sigma}\right)}{\left|I_{\sigma}\right|} .
$$

Then for almost every $x \in[0,1]$ we denote by $\sigma(x)$ the branch landing at it and by $\sigma_{n}(x)$ the $n$-th vertex on this branch. Then for a. e. $x$

$$
\liminf _{n \rightarrow \infty} f\left(\sigma_{n}(x)\right) \geq \lim _{n \rightarrow \infty}\left\langle\phi^{2}\right\rangle_{I_{\sigma}} \cdot \frac{\mu\left(I_{\sigma}\right)}{\left|I_{\sigma}\right|}=\phi^{2}(x) w(x) .
$$

Recall that $I=I_{\sigma_{0}}=[0,1]$. It is time to use Lemma 8 , the last inequalities, and Fatou's lemma (which is $\int \liminf f_{n} f_{n} \leq \liminf _{n} \int f_{n}$ for a sequence of non-negative functions $f_{n}$ ):

$$
\frac{1}{\left|I_{\sigma_{0}}\right|} \int_{I_{\sigma_{0}}} \phi^{2}(x) w(x) d x+\frac{1}{\left|I_{\sigma_{0}}\right|} \sum_{\sigma \in \mathcal{T}}\langle\phi\rangle_{I_{\sigma}}^{2} \alpha_{I_{\sigma}} \leq B\left(x_{1}, x_{2}, x_{3}\right) .
$$

We used here our lemma and (8.12).

But inequality (8.13) proves that $\mathbf{B}(x ; 0,1) \leq B(x ; 0,1)$. In fact, in the left hand side of (8.13) we have arbitrary $\phi$ satisfying (8.10) and arbitrary numbers $\alpha$. satisfying (8.11). Function $\mathbf{B}\left(x_{1}, x_{2}, x_{3}\right)$ by definition is the supremum of such sums over all such functions and collections of numbers. Therefore, inequality $B_{c}(x) \geq \mathbf{B}_{c}(x)$ is completely proved and so is the theorem.

\section{9. $B_{c}(x) \leq \mathbf{B}_{c}(x)$. EXtremal Sequences.}

Theorem 9. Let $c \in(0,1]$. In $\left\{x_{1}^{2} \leq x_{2}, 1-c<x_{3} \leq 1\right.$ we have $\mathbf{B}_{c} \geq B_{c}$.

Proof. Below $I_{0}=[0,1]$. We consider first only $c=1$ and $x=\left(x_{1}, x_{2}, 1\right)$ on the "upper lid" of $\Omega$. We want to fix a large integer $n$ and to construct function $\phi_{n}$ and sequence $\left\{\alpha_{\ell}^{n}\right\}_{\ell \in D\left(I_{0}\right)}$ in such a way that the sum

$$
\sum_{\ell \in D\left(I_{0}\right)}\left\langle\phi_{n}\right\rangle_{\ell}^{2} \alpha_{\ell}^{n}>B\left(x_{1}, x_{2}, 1\right)-\varepsilon_{n}, \quad \varepsilon_{n} \rightarrow 0 .
$$

where

$$
\left\langle\phi_{n}\right\rangle_{I_{0}}=x_{1}
$$


and

$$
\left\langle\phi_{n}^{2}\right\rangle_{I_{0}}=x_{2}
$$

Fix a large integer $n$ and split $I_{0}=[0,1]$ into the union of $n+1$ intervals $I_{k}=$ $\left[2^{-k}, 2^{-k+1}\right], 1 \leq k \leq n$, and $J=\left[0,2^{-n}\right]$. Maps $m_{k}(x)=2^{k} x-1$ map $I_{k}, 1 \leq k \leq$ $n$ onto $I_{0}$. Consider preimages of $I_{s}, s=1, \ldots, n$, under $m_{k}, k=1, \ldots, n$. We obtain $I_{k s}$. Then $m_{s} \circ m_{k}$ maps $I_{k s}$ onto $I_{0}$. We also consider $J_{k}, k=1, \ldots, n$, preimages of $J$ under $m_{k}$. We iterate this procedure obtaining $I_{k_{1} k_{2} \ldots k_{m}}, J_{k_{1} k_{2} \ldots k_{m-1}}$. Put $\alpha_{I_{k}}^{n}:=2^{-k} \cdot 2^{-n}=\left|J_{k}\right|, 0 \leq k \leq n$. We put $\alpha_{k_{1} k_{2} \ldots k_{m}}^{n}=\left|J_{k_{1} k_{2} \ldots k_{m}}\right|$. For all other dyadic intervals we put $\alpha_{I}=0$. We call $J$ the 0 generation, and all $J_{k_{1} k_{2} \ldots k_{m}}$ the $m$-th generation. Obviously sum of lengths of all generations of $J$ 's is equal to 1 . There for we have proved

$$
\sum_{\ell \in D\left(I_{0}\right)} \alpha_{\ell}=1
$$

Moreover, it is easy to see that all $J$ 's are disjoint (except may be the end-points) so

$$
\sum_{\ell \in D\left(I_{0}\right), \ell \subset I} \alpha_{\ell} \leq|I| \forall I \in D\left(I_{0}\right)
$$

Now we are going to define $\phi_{n}$.

$$
\phi_{n}(x)=\left\{\begin{array}{l}
c_{n} x_{1} \quad x \in J \\
\phi_{n}\left(m_{k}(x)\right) \quad x \in I_{k} \quad k=2, \ldots, n \\
d_{n} \phi_{n}\left(m_{1}(x)\right) \quad x \in I_{1} .
\end{array}\right.
$$

Here $c_{n}$ and $d_{n}$ are constants which we will define now. First of all notice that this recursive definition of $\phi_{n}$ really defines it a.e. (as long as $c_{n}, d_{n}$ are prescribed) just because we have it already defined on the 0 generation of $J$ 's (that is on $J$ itself). But then it is defined on $m_{k}$-pre-images of $J$ (so on the first generation of $J$ 's), but then it is defined on pre-images of pre-images (second generation of $J$ 's), et cetera... But The union of all generations of $J$ 's gives us $I_{0}$ up to a set of zero Lebesgue measure.

We define $c_{n}, d_{n}$ from requirements (9.1), (9.2):

$$
\begin{gathered}
\frac{1}{2^{n}} c_{n} x_{1}+\left(1-\frac{1}{2^{n}}-\frac{1}{2}\right) x_{1}+\frac{1}{2} d_{n} x_{1}=x_{1} \\
\frac{1}{2^{n}} c_{n}^{2} x_{2}+\left(1-\frac{1}{2^{n}}-\frac{1}{2}\right) x_{2}+\frac{1}{2} d_{n}^{2} x_{2}=x_{2} .
\end{gathered}
$$


Then we get (using our notations $s=x_{1}^{2} / x_{2}$ and choosing proper root of quadratic equation)

$$
c_{n}=\frac{\left(2^{n-1}+1\right) 1 / s-\sqrt{2^{n-1}\left(2^{n-1}+1\right)\left(x_{1}^{2}-1 / s\right)}}{2^{n-1}+1 / s} \rightarrow 1 / s-\sqrt{1 / s^{2}-1 / s} .
$$

Lemma 10. We have

$$
\phi(x) c_{n} \chi_{J_{k_{1} \ldots k_{m}}} \leq c_{n}\left\langle\phi_{n}\right\rangle_{I_{k_{1} \ldots k_{m}}}, \quad \forall x \in J_{k_{1} \ldots k_{m}},
$$

where empty sequence $k_{1} \ldots k_{m}$ corresponds to $I_{0}$ and $J$ correspondingly.

Proof. Let us start with the empty sequence. Then we should check that

$$
\phi(x) \leq c_{n}\left\langle\phi_{n}\right\rangle_{I_{0}}, \forall x \in J
$$

But the average over $I_{0}$ is $x_{1}$, and $\phi(x)=c_{n} x_{1}$ on $J$ by definition. The rest of the lemma follows from the self-similarity of $\phi_{n}$, in fact, it is either the same function $\phi_{n}$ "pre-shrunk" to a smaller interval, or it is a fixed multiple of this. So we are done.

Let us now square (9.5) and integrate it. Then sum over all $J$ 's of all generations. We will get from (9.4)

$$
\sum_{\ell \in D\left(I_{0}\right)}\left\langle\phi_{n}\right\rangle^{2} \alpha_{\ell}^{n} \geq \frac{x_{2}}{c_{n}^{2}}=x_{1}^{2} \frac{1 / s}{\left(1 / s-\sqrt{1 / s^{2}-1 / s}\right)^{2}}-\varepsilon_{n} .
$$

But the first term is

$$
\begin{aligned}
& \frac{x_{1}^{2}}{(\sqrt{1 / s}-\sqrt{1 / s-1})^{2}}=x_{1}^{2}(\sqrt{1 / s}+\sqrt{1 / s-1})^{2} \\
= & x_{1}^{2}\left(\sqrt{\frac{x_{2}}{x_{1}^{2}}}-\sqrt{\frac{x_{2}}{x_{1}^{2}}-1}\right)^{2}=\left(\sqrt{x_{2}}+\sqrt{x_{2}-x_{1}^{2}}\right)^{2} .
\end{aligned}
$$

We finally get

$$
\sum_{\ell \in D\left(I_{0}\right)}\left\langle\phi_{n}\right\rangle^{2} \alpha_{\ell}^{n} \geq\left(\sqrt{x_{2}}+\sqrt{x_{2}-x_{1}^{2}}\right)^{2}-\varepsilon_{n} .
$$

Let us now look at formula (4.56) with $x_{3}=1$ and plug $a$ from (4.52) with $x_{3}=1$. Readily, (4.52) with $x_{3}=1$ becomes $\frac{1+4 a}{(1+2 a)^{2}}=\frac{x_{1}^{2}}{x_{2}}$. From here and from negativity of $a$ we obtain

$$
\frac{1}{1+2 a}=1+\sqrt{\frac{x_{2}-x_{1}^{2}}{x_{2}}}
$$


But formula (4.56) with $x_{3}=1$ (and $c=1$ ) gives

$$
B\left(x_{1}, x_{2}, 1\right)=\left(\frac{1}{1+2 a}\right)^{2} x_{2}=\left(1+\sqrt{\frac{x_{2}-x_{1}^{2}}{x_{2}}}\right)^{2} x_{2}=\left(\sqrt{x_{2}}+\sqrt{x_{2}-x_{1}^{2}}\right)^{2} .
$$

Using (9.6) we finally we get

$$
\sum_{\ell \in D\left(I_{0}\right)}\left\langle\phi_{n}\right\rangle^{2} \alpha_{\ell}^{n} \geq B\left(x_{1}, x_{2}, 1\right)-\varepsilon_{n},
$$

and our theorem is proved for $x_{3}=1, c=1$.

If $c=1$ but $0<x_{3}<1$, then point $x=\left(x_{1}, x_{2}, x_{3}\right) \in \Omega$ lies on a certain line $L_{t_{1}, t_{2}}$. Function $B$ is linear on this line. So combining constant function and function $\phi_{n}$ we will obtain the general inequality.

For $c \in(0,1)$ the proof is actually exactly the same.

9.1. Geometry of foliation: explanation of the choice of $\phi_{n}$ in (9.3). This section contains a heuristic explanation why function $\phi_{n}$ was chosen in this form in (9.3).

The main point here is a certain geometric observation concerning the foliation of the domain $\Omega$ by lines $L_{t_{1} t_{2}}$. Consider the foliation of the upper lid $\left\{X_{3}=1\right\} \cap \Omega$ by parabolas $P_{A}:=\left\{X_{2}=A X_{1}^{2}, X_{3}=1\right\}, A \geq 1$. Then we have the following easy geometric observation.

Theorem 11. Let $L_{t_{1}, t_{2}}$ intersects the upper lid at the point $\left(u_{1}, u_{2}, 1\right)$ and let $\left(u_{1}, u_{2}, 1\right) \in P_{A}$. Then the projection of the line $L_{t_{1} t_{2}}$ onto the upper lid is tangent to $P_{A}$ at point $\left(u_{1}, u_{2}, 1\right)$.

Proof. Fix $t_{1}, t_{2}$, which fixes $a=a\left(t_{2}\right)$ and consider formulae (4.48) that give us $L_{t_{1} t_{2}}$. Then we can compute the slope of the projection of $L_{t_{1} t_{2}}$ on plane $\left(X_{1}, X_{2}\right)$. In fact from (4.48)

$$
\frac{\partial X_{1}}{\partial X_{3}}=-2 t_{1} a^{2}, \frac{\partial X_{2}}{\partial X_{3}}=\frac{4 t_{1}^{2} a^{3}(1-2 a)^{2}}{1-4 a} .
$$

Therefore, the slope of this projection is the ratio of these quantities:

$$
-\frac{2 t_{1} a(1-2 a)^{2}}{1-4 a}
$$

On the other hand the point of intersection of $L_{t_{1} t_{2}}$ wit the plane $\left\{X_{3}=1\right\}$ is (again see (4.48))

$$
U=\left(u_{1}, u_{2}, 1\right), \text { where } u_{1}=-t_{1} a, u_{2}=t_{1}^{2} a^{2}(1-2 a)^{2} .
$$


Therefore, point $U$ lies on parabola $v_{3}=1, v_{2}=\frac{(1-2 a)^{2}}{1-4 a} \cdot v_{1}^{2}$, and, hence, the slope of this parabola at $U$ is

$$
\frac{2(1-2 a)^{2}}{1-4 a} \cdot u_{1}
$$

Now plug $u_{1}=-t_{1} a$ into (9.9) to see that it is equal to the formula for slope in (9.8). We are done.

Let us see what influence this theorem has on the choice of $\phi_{n}$.

Given $L=L_{t_{1} t_{2}}$ intersecting the upper lid at $u=\left(u_{1}, u_{2}, 1\right)$. To construct $\phi$ corresponding to $u$ we make an infinitesimally small jumps $u+\varepsilon e, u-\varepsilon e$ along $L$ (here $e$ is a unit vector parallel to $L$ and with positive third coordinate). If we would know how to build the extremal functions $\phi_{ \pm}$for $u \pm \varepsilon e$ we would just put the one for $u+\varepsilon e$ on the right half $I_{+}$of $I$ and the one for $u-\varepsilon e$ on the left half $I_{-}$ of $I$. The one for $u+\varepsilon e$ is definitely unknown. But the one for $u-\varepsilon e$ is "known" in the sense that we can jump from it to $u-2 \varepsilon e=u-\varepsilon e-\varepsilon e$ and $u=u-\varepsilon e+\varepsilon e$. Then we can restart the procedure again. Thus, the explanation for the self-similar structure of $\phi_{n}$ and for the second line of (9.3).

Notice that after several jumps along $L$ we will find ourselves at the end-point $u-n \varepsilon e$ of $L$ where $X_{3}=0, X_{2}=X_{1}^{2}$ (especially if we choose dyadic number $\varepsilon$ ). At this moment we know that extremal function is just constant $X_{1}=: c_{n} u_{1}$. This is the explanation for the first line in the definition of $\phi_{n}$.

To explain $d_{n}$ let us recall that we do not yet know how to build the extremal function for the point $u+\varepsilon e$. Moreover, we cannot do that. This point is outside of $\Omega$. So here is heuristics. Instead of jumping into $u+\varepsilon e$ let us jump to point $p_{\varepsilon}=$ projection of $u+\varepsilon e$ onto the upper lid. We know by Theorem 11 that $p_{\varepsilon}$ lies on the tangent line to parabola $P_{A}: x_{2}=A x_{1}^{2}$ at point $u$. So, in a sense, we can think that $p_{\varepsilon}$ lies on $P_{A}$ (because $\varepsilon$ is very small). Or rather we can choose $U=p_{\varepsilon}+O\left(\varepsilon^{2}\right)$ such that $U \in P_{A}$. Let $U=\left(U_{1}, U_{2}, 1\right)$.

Now we need to understand the following: suppose we know an almost extremal function for $u$ (our future $\phi_{n}$ ). Do we know it for $U$ then? The answer is yes, because if we know the extremal function for one point of parabola than the extremal function for another point of the same parabola is acquired just by multiplication of the first function on a suitable constant.

This is the explanation for the third line of (9.3). 


\section{Step I for Carleson embedding theorems in $L^{p}$ With $p>1$}

Now, we briefly consider how to find the Bellman function for the Carleson embedding operator acting on arbitrary $L^{p}$. We shell follow the same scheme and we shall start with an evident definition of the Bellman function.

$$
\begin{gathered}
\mathbf{B}_{\max }\left(x_{1}, x_{2}, x_{3} ; m, M\right):=\sup \left\{\int_{I}|\phi(t)|^{p} d \mu(t)+\sum_{J \in D(I)}\left|\langle\phi\rangle_{J}\right|^{p} \alpha_{J}:\right. \\
\langle\phi\rangle_{I}=x_{1}, \quad\left\langle|\phi|^{p}\right\rangle_{I}=x_{2}, \quad \frac{1}{|I|}\left(\mu(I)+\sum_{J \in D(I)} \alpha_{J}\right)=x_{3}, \\
\left.m|J| \leq \mu(J)+\sum_{\ell \in D(J)} \alpha_{\ell} \leq M|J| \quad \forall J \in D(I)\right\}, \\
\mathbf{B}_{\min }\left(x_{1}, x_{2}, x_{3} ; m, M\right):=\inf \left\{\int_{I}|\phi(t)|^{p} d \mu(t)+\sum_{J \in D(I)}\left|\langle\phi\rangle_{J}\right|^{p} \alpha_{J}:\right. \\
\langle\phi\rangle_{I}=x_{1}, \quad\left\langle|\phi|^{p}\right\rangle_{I}=x_{2}, \quad \frac{1}{|I|}\left(\mu(I)+\sum_{J \in D(I)} \alpha_{J}\right)=x_{3}, \\
\left.m|J| \leq \mu(J)+\sum_{\ell \in D(J)} \alpha_{\ell} \leq M|J| \quad \forall J \in D(I)\right\} .
\end{gathered}
$$

Functions $\mathbf{B}$ are defined in

$$
\Omega:=\left\{x=\left(x_{1}, x_{2}, x_{3}\right): x_{1}^{p} \leq x_{2}, m \leq x_{3} \leq M\right\} .
$$

As before, the Bellman functions $\mathbf{B}$ do not depend on the interval $I$. The difference is that now we consider non-negative test functions only.

From the definition of function $\mathbf{B}$ above we can immediately see that it satisfies the inequality (just concavity or convexity)

$$
\begin{aligned}
& \mathbf{B}_{\max }(x)-\frac{1}{2}\left(\mathbf{B}_{\max }\left(x^{-}\right)+\mathbf{B}_{\max }\left(x^{+}\right)\right) \geq 0, \quad \forall x^{ \pm} \in \Omega, \quad x=\frac{1}{2}\left(x^{+}+x^{-}\right) . \\
& \mathbf{B}_{\min }(x)-\frac{1}{2}\left(\mathbf{B}_{\min }\left(x^{-}\right)+\mathbf{B}_{\min }\left(x^{+}\right)\right) \leq 0, \quad \forall x^{ \pm} \in \Omega, \quad x=\frac{1}{2}\left(x^{+}+x^{-}\right) .
\end{aligned}
$$

Note that $\Omega$ (see (10.3) $)$ is convex and for all $x^{ \pm} \in \Omega$ we have $x \in \Omega$.

The boundary conditions also follow from the definition: 


$$
\begin{gathered}
\mathbf{B}\left(x_{1},\left|x_{1}\right|^{p}, x_{3}\right)=\left|x_{1}\right|^{p} x_{3} . \\
\frac{\partial \mathbf{B}}{\partial x_{3}}\left(x_{1}, x_{2}, M ; m, M\right)=\left|x_{1}\right|^{p} .
\end{gathered}
$$

It is equally easy to see that homogeneity condition holds

$$
\mathbf{B}\left(t x_{1}, t^{p} x_{2}, x_{3}\right)=t^{p} \mathbf{B}\left(x_{1}, x_{2}, x_{3}\right), \quad t>0, .
$$

As before, we are looking for a function $B$ satisfying the following degeneration condition:

$$
\operatorname{det}\left(d^{2} B\right)=\operatorname{det}\left(\begin{array}{lll}
B_{x_{1} x_{1}} & B_{x_{1} x_{2}} & B_{x_{1} x_{3}} \\
B_{x_{2} x_{1}} & B_{x_{2} x_{2}} & B_{x_{2} x_{3}} \\
B_{x_{3} x_{1}} & B_{x_{3} x_{2}} & B_{x_{3} x_{3}}
\end{array}\right)=0, \quad \forall x \in \Omega
$$

Lemma 12. There is a simple algorithm to find the function $B(x ; m, M)$ that solves Monge-Ampère equation (10.9) in the domain (10.3) with boundary condition (10.6)-(10.7) and homogeneity condition (10.8).

Proof. Again we look for a solution $B$ of the form

$$
B\left(x_{1}, x_{2}, x_{3}\right)=t_{1} \cdot x_{1}+t_{2} \cdot x_{2}+t_{3} \cdot x_{3}+t_{0},
$$

where $t_{i}=\frac{\partial B}{\partial x_{i}}, i=1,2,3$. The integral curves of the vector field $\Theta$ such that $d^{2} B(x) \Theta(x)=0$ are again segments of straight lines

$$
x_{1} d t_{1}+x_{2} d t_{2}+x_{3} d t_{3}+d t_{0}=0 .
$$

Each line is given by fixing two free parameters, for example $t_{1}$ and $t_{2}$. Then we can rewrite equation (10.11) defining lines $L_{t_{1}, t_{2}}$ foliating our domain in the following form:

$$
\left\{\begin{array}{l}
x_{1}+\frac{\partial t_{3}}{\partial t_{1}} \cdot x_{3}+\frac{\partial t_{0}}{\partial t_{1}}=0 \\
x_{2}+\frac{\partial t_{3}}{\partial t_{2}} \cdot x_{3}+\frac{\partial t_{0}}{\partial t_{2}}=0
\end{array}\right.
$$

Differentiating (10.8) in $t$ and setting $t=1$ we get

$$
x_{1} t_{1}+p x_{2} t_{2}=p B \text {. }
$$

Whence,

$$
(p-1) t_{1} x_{1}+p t_{3} x_{3}+p t_{0}=0 .
$$


If we subtract this equation from the first equation of (10.12) multiplied by $(p-1) t_{1}$, then we get

$$
\left(p t_{3}-(p-1) t_{1} \frac{\partial t_{3}}{\partial t_{1}}\right) x_{3}+\left(p t_{0}-(p-1) t_{1} \frac{\partial t_{0}}{\partial t_{1}}\right)=0
$$

for every $x_{3}$. If we introduce the dual exponent $q=\frac{p}{p-1}$, then the latter equation can be rewritten as follows

$$
\left(q t_{3}-t_{1} \frac{\partial t_{3}}{\partial t_{1}}\right) x_{3}+\left(q t_{0}-t_{1} \frac{\partial t_{0}}{\partial t_{1}}\right)=0 .
$$

Hence

$$
\left\{\begin{array}{l}
q t_{3}-t_{1} \frac{\partial t_{3}}{\partial t_{1}}=0 \\
q t_{0}-t_{1} \frac{\partial t_{0}}{\partial t_{1}}=0
\end{array}\right.
$$

From these "PDE" we easily write down

$$
\left\{\begin{array}{l}
t_{3}=A\left(t_{2}\right)\left|t_{1}\right|^{q} \\
t_{0}=D\left(t_{2}\right)\left|t_{1}\right|^{q} .
\end{array}\right.
$$

Then the equations of the extremal lines $L_{t_{1}, t_{2}}$ can be rewritten in the form

$$
\left\{\begin{array}{l}
x_{1}+q\left|t_{1}\right|^{q-2} t_{1} A x_{3}+q\left|t_{1}\right|^{q-2} t_{1} D=0 \\
x_{2}+\left|t_{1}\right|^{q} A^{\prime} x_{3}+\left|t_{1}\right|^{q} D^{\prime}=0
\end{array}\right.
$$

As before, we assume that our extremal line $L_{t_{1}, t_{2}}$ intersect the boundary $\partial \Omega$ in a point $\zeta=\zeta\left(t_{1}, t_{2}\right)$ on the "upper lid" $x_{3}=M$ and in a point $\xi=\xi\left(t_{1}, t_{2}\right)$ on the "side" $x_{2}=\left|x_{1}\right|^{p}$. Then we have two pairs of equations (10.17) asserting that our points are on the line $L_{t_{1}, t_{2}}$

$$
\begin{array}{r}
\zeta_{1}+q\left|t_{1}\right|^{q-2} t_{1} A M+q\left|t_{1}\right|^{q-1} t_{1} D=0 \\
\zeta_{2}+\left|t_{1}\right|^{q} A^{\prime} M+\left|t_{1}\right|^{q} D^{\prime}=0 \\
\xi_{1}+q\left|t_{1}\right|^{q-2} t_{1} A \xi_{3}+q\left|t_{1}\right|^{q-2} t_{1} D=0 \\
\left|\xi_{1}\right|^{p}+\left|t_{1}\right|^{q} A^{\prime} \xi_{3}+\left|t_{1}\right|^{q} D^{\prime}=0
\end{array}
$$

and two boundary conditions (10.6) and (10.7)

$$
\begin{aligned}
\frac{1}{p} \xi_{1} t_{1}+\left|\xi_{1}\right|^{p} t_{2} & =\left|\xi_{1}\right|^{p} \xi_{3} \\
A\left|t_{1}\right|^{q} & =\left|\zeta_{1}\right|^{p} .
\end{aligned}
$$


If we take $\zeta_{1}$ from (10.18) and plug into (10.23) we get

$$
A=q^{p}|A M+D|^{p} .
$$

So, introducing a new function of $t_{2}$

$$
a=q(A M+D)
$$

we can express both functions $A$ and $D$ in terms of $a$ : (10.24) yields

$$
A=|a|^{p}
$$

and directly from the definition of $a(10.25)$ we get

$$
D=\frac{1}{q} a-M|a|^{p} .
$$

In result equations (10.20), (10.21), and (10.22) can be rewritten as follows

$$
\begin{aligned}
\xi_{1}+\left|t_{1}\right|^{q-2} t_{1} a\left(1-q a|a|^{p-2}\left(M-\xi_{3}\right)\right) & =0 \\
\left|\xi_{1}\right|^{p}+\frac{p}{q}\left|t_{1}\right|^{q} a^{\prime}\left(\frac{1}{p}-q a|a|^{p-2}\left(M-\xi_{3}\right)\right) & =0 \\
\frac{1}{p} \xi_{1} t_{1}-\left|\xi_{1}\right|^{p}\left(\xi_{3}-t_{2}\right) & =0 .
\end{aligned}
$$

As before, we introduce the following new function

$$
\eta=1-q a|a|^{p-2}\left(M-\xi_{3}\right),
$$

and then equations (10.28) and (10.29) turn into

$$
\begin{aligned}
\xi_{1}+\left|t_{1}\right|^{q-2} t_{1} a \eta & =0 \\
\left|\xi_{1}\right|^{p}+\frac{p}{q}\left|t_{1}\right|^{q} a^{\prime}\left(\eta-\frac{1}{q}\right) & =0,
\end{aligned}
$$

whence

$$
a^{\prime}\left(\eta-\frac{1}{q}\right)+\frac{q}{p}|a|^{p}|\eta|^{p}=0 .
$$

Since $a$ does not depend on $t_{1}$, the function $\eta$ (as a solution of this equation) also depends only on $t_{2}$.

On the one side, from (10.31) we have

$$
\xi_{3}=M-\frac{1-\eta}{q a|a|^{p-2}},
$$

on the other side (10.30) together with (10.32) yield

$$
\xi_{3}=t_{2}+\frac{\xi_{1} t_{1}}{p\left|\xi_{1}\right|^{p}}=t_{2}-\frac{a \eta}{p|a|^{p}|\eta|^{p}} .
$$


So, excluding $x_{3}$ we get

$$
t_{2}-\frac{a \eta}{p|a|^{p}|\eta|^{p}}=M-\frac{1-\eta}{q a|a|^{p-2}} .
$$

Differentiating in $t_{2}$ the latter equation we obtain

$$
1+\frac{a^{\prime} \eta+\eta^{\prime} a}{q|a|^{p}|\eta|^{p}}=\frac{\eta^{\prime}}{q a|a|^{p-2}}+\frac{p-1}{q} \cdot \frac{(1-\eta) a^{\prime}}{|a|^{p}}
$$

and then we multiply this equality by the common denominator and replace it using (10.34) by $-p a^{\prime}\left(\eta-\frac{1}{q}\right)$ :

$$
-p a^{\prime}\left(\eta-\frac{1}{q}\right)+a^{\prime} \eta+\eta^{\prime} a=\eta^{\prime} a|\eta|^{p}+(p-1)(1-\eta) a^{\prime}|\eta|^{p},
$$

or

$$
(p-1)\left(|\eta|^{p}-1\right)(\eta-1) a^{\prime}=a\left(|\eta|^{p}-1\right) \eta^{\prime} .
$$

Thus, we come to the following equation

$$
(p-1)(\eta-1) a^{\prime}=a \eta^{\prime},
$$

which implies $\eta=1+C|a|^{p}$. Then (10.35) yields

$$
\xi_{3}\left(t_{2}\right)=M+\frac{C|a|^{p-1}}{q a|a|^{p-2}}=M+\frac{C}{q} \operatorname{sign} a=: c .
$$

The latter expression is a constant, because $\operatorname{sign} a$ can take the only value due to $\xi_{3} \leq M$. As before, the natural choice of the constant $c$ is $c=m$.

Recall that we have the following expression for $B$ (see (10.13))

$$
B=\frac{1}{p} t_{1} x_{1}+t_{2} x_{2}
$$

Thus, to find an expression for $B$ we need to find $t_{1}$ and $t_{2}$ as functions of a point $x$ running over our domain $\Omega$.

From (10.31) we have

$$
\eta=1-q a|a|^{p-2}(M-m)
$$

hence $(10.36)$ yields

$$
t_{2}=m+\frac{a \eta}{p|a \eta|^{p}}=m+\frac{a-q(M-m)|a|^{p}}{\left.\left.p|a-q(M-m)| a\right|^{p}\right|^{p}} .
$$

This gives us the desired $a$ as a function of $t_{2}$, but it is clear that $a$ is more convenient parameter than $t_{2}$. We shall express all other functions of $t_{2}$ in terms 
of $a$ and look for $a$ as a function of $x \in \Omega$. To this aim we return to the equations of the extremal lines (10.17) rewriting them in terms of $a$. From (10.41) we have

$$
\frac{d t_{2}}{d a}=-\frac{1}{q|a \eta|^{p}} \cdot \frac{d}{d a}(a \eta)=-\frac{1-p q(M-m)|a|^{p-2} a}{\left.\left.q|a-q(M-m)| a\right|^{p}\right|^{p}}
$$

therefore

$$
a^{\prime}=-\frac{\left.\left.q a|a-q(M-m)| a\right|^{p}\right|^{p}}{a-p q(M-m)|a|^{p}}
$$

and

$$
\begin{aligned}
A=|a|^{p}, & A^{\prime}=p|a|^{p-2} a a^{\prime}=-p q|a|^{p} \frac{\left.\left.|a-q(M-m)| a\right|^{p}\right|^{p}}{a-p q(M-m)|a|^{p}} \\
D= & \frac{1}{q} a-M|a|^{p}, D^{\prime}=\frac{1}{q}\left(1-p q M|a|^{p-2} a\right) a^{\prime}= \\
& -\frac{\left.\left.\left(a-p q M|a|^{p}\right)|a-q(M-m)| a\right|^{p}\right|^{p}}{a-p q(M-m)|a|^{p}}
\end{aligned}
$$

Now equation (10.17) of the line $L_{t_{1}, t_{2}}$ becomes

$$
\left\{\begin{array}{l}
x_{1}=-\left|t_{1}\right|^{q-2} t_{1}\left(a-q\left(M-x_{3}\right)|a|^{p}\right), \\
x_{2}=\left|t_{1}\right|^{q} \frac{\left.\left.|a-q(M-m)| a\right|^{p}\right|^{p}\left(a-p q\left(M-x_{3}\right)|a|^{p}\right)}{a-p q(M-m)|a|^{p}} .
\end{array}\right.
$$

To get a more symmetrical expression, as before, we take a more geometrical parameter $\xi_{1}$ instead of $t_{1}$, where $\xi_{1}$ is the first coordinate of $\xi=\left(\xi_{1},\left|\xi_{1}\right|^{p}, m\right)$, the point where the side surface $\left\{x_{2}=\left|x_{1}\right|^{p}\right\}$ is intersected by our extremal line. In term of $a$ and $\xi_{1}$ equations (10.42) turns into

$$
\left\{\begin{array}{l}
x_{1}=\frac{a-q\left(M-x_{3}\right)|a|^{p}}{a-q(M-m)|a|^{p}} \xi_{1}, \\
x_{2}=\frac{a-p q\left(M-x_{3}\right)|a|^{p}}{a-p q(M-m)|a|^{p}}\left|\xi_{1}\right|^{p} .
\end{array}\right.
$$

These equations immediately supply us with an expression for $a$, namely and $a=a(x)=$ is a root of the following equation

$$
s:=\frac{\left|x_{1}\right|^{p}}{x_{2}}=\left|\frac{a-q\left(M-x_{3}\right)|a|^{p}}{a-q(M-m)|a|^{p}}\right|^{p} \frac{a-p q(M-m)|a|^{p}}{a-p q\left(M-x_{3}\right)|a|^{p}} .
$$

To determine which of possible roots of the equation (10.44) gives us the desired value of $a$, we investigate the above function $s=s(a)$ defined by (10.44) as a function of the parameter $a$, all other parameters assuming to be fixed. 
First of all we note that the extremal line (10.43) intersects the plane $\left\{x_{3}=M\right\}$ at the point

$$
\zeta=\left(\zeta_{1}, \zeta_{2}, M\right), \quad \zeta_{1}=\frac{a \xi_{1}}{a-p q\left(M-x_{3}\right)|a|^{p}}, \quad \zeta_{2}=\frac{a\left|\xi_{1}\right|^{p}}{a-p q\left(M-x_{3}\right)|a|^{p}} .
$$

Since $\zeta_{2} \geq 0$, we have the first restriction for $a$ :

$$
a<\left(\frac{1}{p q(M-m)}\right)^{q-1} .
$$

The behavior of $s(a)$ on the semi-axis (10.46) is the same for all values of other parameters: on the negative half-line it monotonously increases from $\left(\frac{M-x_{3}}{M-m}\right)^{p-1}$ till 1 , and on the interval $\left[0,\left(\frac{1}{p q(M-m)}\right)^{q-1}\right]$ it monotonously decreases from 1 to 0 . Therefore, for a given $s, 0 \leq s \leq 1$, we have one or two solutions of (10.44) satisfying (10.46): for all $s$ there exists a positive solution $a=a^{+}$and for $s>$ $\left(\frac{M-x_{3}}{M-m}\right)^{p-1}$ there exists a negative solution $a=a^{-}$as well. We already know that the solution $a=a^{+}$corresponds to the function $\mathbf{B}_{\max }$, and the solution $a=a^{-}$ corresponds to the function $\mathbf{B}_{\min }$.

To write down the expression for $B$ we use (10.13), then we express $x_{1}$ using the first equation of (10.42), and by means of the second equation of (10.42) we replace the expression of $\left|t_{1}\right|^{q}$. For $t_{2}$ we use (10.41). In result we obtain

$$
\begin{gathered}
B=\frac{1}{p} x_{1} t_{1}+x_{2} t_{2}=-\frac{1}{p}\left|t_{1}\right|^{q}\left(a-q\left(M-x_{3}\right)|a|^{p}\right)+x_{2} t_{2}= \\
-\frac{\left(a-q\left(M-x_{3}\right)|a|^{p}\right)\left(a-p q(M-m)|a|^{p}\right)}{\left.\left.p|a-q(M-m)| a\right|^{p}\right|^{p}\left(a-p q\left(M-x_{3}\right)|a|^{p}\right)} x_{2}+x_{2}\left(m+\frac{a-q(M-m)|a|^{p}}{\left.\left.p|a-q(M-m)| a\right|^{p}\right|^{p}}\right)= \\
\frac{a|a|^{p}\left(x_{3}-m\right) x_{2}}{\left.\left.|a-q(M-m)| a\right|^{p}\right|^{p}\left(a-p q\left(M-x_{3}\right)|a|^{p}\right)}+m x_{2}= \\
\frac{\left(x_{3}-m\right) x_{2}}{\left.\left.|1-q(M-m) a| a\right|^{p-2}\right|^{p}\left(1-p q\left(M-x_{3}\right) a|a|^{p-2}\right)}+m x_{2} .
\end{gathered}
$$

As it was already mentioned, here we choose the solution $a=a^{+}$of (10.44) for $B_{\max }$ and $a=a^{-}$for $B_{\min }$.

Theorem 13. Consider

$$
B\left(x_{1}, x_{2}, x_{3} ; m, M\right)=\frac{\left(x_{3}-m\right) x_{2}}{[1-2 a(M-m)]^{2}\left[1-4 a\left(M-x_{3}\right)\right]}+m x_{2} .
$$


We shall denote this function by $B_{\max }$ if $a=a^{+}(x)$ is the unique positive solution of the equation

$$
\frac{\left|x_{1}\right|^{p}}{x_{2}}=\left|\frac{a-q\left(M-x_{3}\right)|a|^{p}}{a-q(M-m)|a|^{p}}\right|^{p} \frac{a-p q(M-m)|a|^{p}}{a-p q\left(M-x_{3}\right)|a|^{p}}
$$

from the interval $\left[0,\left(\frac{1}{p q(M-m)}\right)^{q-1}\right]$, and this function will be denoted by $B_{\min }$ if $a=a^{-}(x)$ is the unique negative solution of the same equation. The domain of $B_{\max }$ is

$$
\operatorname{Dom}\left(B_{\max }\right)=\left\{x: x_{2} \geq\left|x_{1}\right|^{p}, m \leq x_{3} \leq M\right\},
$$

the domain of $B_{\min }$ is

$$
\operatorname{Dom}\left(B_{\min }\right)=\left\{x:\left|x_{1}\right|^{p} \leq x_{2} \leq\left(\frac{M-m}{M-x_{3}}\right)^{p-1}\left|x_{1}\right|^{p}, m \leq x_{3} \leq M\right\} .
$$

Then

$$
\mathbf{B}_{\text {max }}(x ; m, M)=\left\{\begin{array}{cc}
B_{\max }(x ; m, M) & m<x_{3} \leq M \\
m x_{2} & x_{3}=m .
\end{array}\right.
$$

and

$$
\mathbf{B}_{\text {min }}(x ; m, M)=\left\{\begin{array}{cl}
B_{\min }(x ; m, M) & M-(M-m) \frac{\left|x_{1}\right|^{q}}{x_{2}^{q-1}} \leq x_{3} \leq M \\
m x_{2} & m \leq x_{3} \leq M-(M-m) \frac{\left|x_{1}\right|^{q}}{x_{2}^{q-1}} .
\end{array}\right.
$$

The proof of this theorem follows almost verbatim the lines of the proof of our Theorems 3, 4 above. So, we are not going to prove it here. Instead, we wish to show that our results encompass a slightly more general situation than it could have been thought.

\section{TREes.}

As in $[\mathrm{M}]$ we let $\left(X, \mu_{0}\right)$ be a nonatomic probability space. Two measurable subsets $A, B$ of $X$ will be called almost disjoint if $\mu_{0}(A \cap B)=0$. Then we give the following

Definition. A set $\mathcal{T}$ is called a tree if the following conditions are satisfied

- $X \in \mathcal{T}$ and for every $I \in \mathcal{T}$ we have $\mu_{0}(I)>0$. 
- For every $I \in \mathcal{T}$ there corresponds a finite or countable subset $C(I) \subset \mathcal{T}$ containing at least two elements such that:

a) the elements of $C(I)$ are pairwise almost disjoint,

b) $I=\cup_{J \in C(I)} J$.

- $\mathcal{T}=\cup_{m \geq 0} \mathcal{T}_{m}$, where $\mathcal{T}_{0}=\{X\}, \mathcal{T}_{m+1}=\cup_{I \in \mathcal{T}_{m}} C(I)$.

- We have $\lim _{m \rightarrow \infty} \sup _{I \in \mathcal{T}_{m}} \mu_{0}(I)=0$.

For any tree $\mathcal{T}$ we define its exceptional set $E=E(\mathcal{T})$ as

$$
E=\cup_{I \in \mathcal{T}} \cup_{J_{1}, J_{2} \in C(I), J_{1} \neq J_{2}} J_{1} \cap J_{2} .
$$

It is clear that $E$ has $\mu_{0}$ measure zero.

An easy induction shows that each $\mathcal{T}_{m}$ consists of pairwise almost disjoint sets whose union is $X$. Moreover, if $x \in X \backslash E$, then for each $m$ there exists exactly one $I_{m}$ in $\mathcal{T}_{m}$ containing $x$. For every $m>0$ there exists a $J \in \mathcal{T}_{m-1}$ such that $I_{m} \in C(J)$. Then of course $J=I_{m-1}$. Hence the set $A(x)=\{I \in \mathcal{T}: x \in I\}$ forms a chain of nested elements of $\mathcal{T}_{m}, m=0,1, \ldots$ Inclusions are all strict. From this remark it clear that if $I, J \in \mathcal{T}$ and $I \cap J \cap(X \backslash E)$ is nonempty then $I \subset J$ or $J \subset I$. In particular, for any $I, J \in \mathcal{T}$ we have either $\mu_{0}(I \cap J)=0$ or one of them is contained in the other. We denote by $\mathcal{T}(J)$ all elements of $\mathcal{T}$ that are subsets of $J$. Also for any $I \in \mathcal{T}$ put

$$
\langle\phi\rangle_{I}:=\frac{1}{\mu_{0}(I)} \int_{I} \phi d \mu_{0} .
$$

Given any tree $\mathcal{T}$ consider the collection $\alpha=\left\{\alpha_{I}\right\}_{I \in \mathcal{T}}$ of non-negative numbers. Let $\mu$ be another measure on $X$. We can introduce the tree Bellman function:

$$
\begin{gathered}
\mathbf{B}_{C E T}^{\mathcal{T}}\left(x_{1}, x_{2}, x_{3} ; m, M\right):=\sup \left\{\int_{X}|\phi(t)|^{2} d \mu(t)+\sum_{J \in \mathcal{T}}\left|\langle\phi\rangle_{J}\right|^{2} \alpha_{J}:\right. \\
\langle\phi\rangle_{X}=x_{1}, \quad\left\langle\phi^{2}\right\rangle_{X}=x_{2}, \quad\left(\mu(X)+\sum_{J \in \mathcal{T}} \alpha_{J}\right)=x_{3}, \\
\left.m \mu_{0}(J) \leq \mu(J)+\sum_{\ell \in \mathcal{T}(J)} \alpha_{\ell} \leq M \mu_{0}(J) \quad \forall J \in \mathcal{T}\right\} .
\end{gathered}
$$

Independently of the tree the same Theorem 3 holds:

Theorem 14. Consider

$$
B\left(x_{1}, x_{2}, x_{3} ; m, M\right)=\frac{\left(x_{3}-m\right) x_{2}}{[1-2 a(M-m)]^{2}\left[1-4 a\left(M-x_{3}\right)\right]}+m x_{2},
$$


where $a=a(x)$ is the unique solution of the cubic equation

$$
\frac{x_{1}^{2}}{x_{2}}=\left[\frac{1-2 a\left(M-x_{3}\right)}{1-2 a(M-m)}\right]^{2} \frac{1-4 a(M-m)}{1-4 a\left(M-x_{3}\right)}
$$

on the interval $\left[0, \frac{1}{4(M-m)}\right]$. Then

$$
\mathbf{B}_{C E T}^{\mathcal{T}}(x ; m, M)=\left\{\begin{array}{cll}
B(x ; m, M) & x_{1}^{2} \leq x_{2}, & m<x_{3} \leq M \\
m x_{2} & x_{1}^{2} \leq x_{2}, \quad x_{3}=m .
\end{array}\right.
$$

We can introduce the tree Bellman function also for $p \neq 2,1<p<\infty$. Again the result for the trees will not depend on the tree, it coincides with dyadic result. The proofs of $\mathbf{B}_{C E T}^{\mathcal{T}}(x ; m, M) \leq B(x ; m, M)$ is exactly the same as before, the proof of $\mathbf{B}_{C E T}^{\mathcal{T}}(x ; m, M) \geq B(x ; m, M)$ is not too difficult either if one uses the following lemma from $[\mathrm{M}]$.

Lemma 15. For every $I \in \mathcal{T}$ and every $\alpha$ such that $0<\alpha<1$ there exists a subfamily $F(I) \subset \mathcal{T}$ consisiting of pairwise almost disjoint subsets of I such that

$$
\mu_{0}\left(\cup_{J \in F(I)} J\right)=(1-\alpha) \mu_{0}(I) .
$$

\section{REFERENCES}

[M] A. Melas, The Bellman functions of dyadic-like maximal operators and related inequalities, Adv. in Math., Vol. 192 (2005), No. 2, pp. 310-340.

[NT] F. Nazarov, S. Treil, The hunt for Bellman function: applications to estimates of singular integral operators and to other classical problems in harmonic analysis, Algebra i Analiz, Vol. 8 (1997), No. 5, pp. 32-162.

[NTV1] F. Nazarov, S. Treil, A. Volberg, The Bellman functions and two-weight inequalities for Haar multipliers, J. Amer. Math. Soc., 12 no. 4 (1999), 909-928.

[NTV2] F. Nazarov, S. Treil, A. Volberg, Bellman function in Stochastic Optimal Control and Harmonic Analysis ( how our Bellman function got its name), Oper. Theory: Advances and Appl. 129 (2001), 393-424.

[PVo] S. Petermichl, A. Volberg, Heating of the Ahlfors-Beurling operator: weakly quasiregular maps on the plane are quasiregular, Duke Math. J., 112 (2002), no. 2, 281-305.

[SiSt] L. Slavin, A. Stokolos, The maxial operator on $L^{p}\left(\mathbb{R}^{n}\right)$, Preprint, 2006.

[SV] L. Slavin, V. Vasyunin, Sharp results in the integral-form John-Nirenberg inequality, Preprint, 2007; http://arxiv.org/abs/0709.4332.

[V] V. Vasyunin, The sharp constant in the John-Nirenberg inequality, Preprint PDMI no.20, 2003; http://www.pdmi.ras.ru/preprint/2003/index.html.

[Vo] A. Volberg, Bellman approach to some problems in Harmonic Analysis, Seminaire des Equations aux derivées partielles, Ecole Polytechnique, 2002, exposé XX, 1-14. 
Vasily Vasyunin, V. A. Steklov Math. Inst., St Petersburg vasyunin@pdmi.ras.ru

Alexander Volberg, Department of Mathematics, Michigan State University and THE UNIVERSITY OF EDINBURGH volberg@math.msu.edu AND a.volberg@ed.ac.uk 